\title{
Organoborane Acceptor-Substituted Polythiophene via Side-Group Borylation
}

\author{
Haiyan Li, Anand Sundararaman, Krishnan Venkatasubbaiah, and Frieder Jäkle*
}

Department of Chemistry, Rutgers University-Newark, 73 Warren Street, Newark, NJ 07102

\section{Supplementary Material}

Full reference 4g: Yuan, Z.; Entwistle, C. D.; Collings, J. C.; Albesa-Jové, D.; Batsanov, A. S.; Howard, J. A. K.; Kaiser, H. M.; Kaufmann, D. E.; Poon, S.-Y.; Wong, W.-Y.; Jardin, C.; Fathallah, S.; Boucekkine, A.; Halet, J.-F.; Taylor, N. J.; Marder, T. B. Chem. Eur. J. 2006, 12, 2758-2771.

Materials and General Methods. ${ }^{\mathrm{t}} \mathrm{BuLi}\left(1.5 \mathrm{M}\right.$ in hexanes), iodine, $\mathrm{KO} \mathrm{Bu}^{t} \mathrm{Bu}$, and $\mathrm{P}^{\mathrm{t}} \mathrm{Bu}_{3}$ were purchased from Acros, $\mathrm{Me}_{3} \mathrm{SnCl}, \mathrm{Pd}_{2}(\mathrm{dba})_{3}$ from Strem Chemicals, $\mathrm{BBr}_{3}$ from Aldrich, and benzo[a]pyrene from TCI chemicals. $\mathrm{BBr}_{3}$ was distilled under vacuum and all other chemicals were used as received without further purification. 3,3'-Bis-(trimethylsilyl)-5,5'-bithiophene $(>97 \%)^{1}, 5$-methyl-2-trimethylstannylthiophene ${ }^{2}$, and mesitylcopper ${ }^{3}$ (recrystallized from toluene) were prepared according to literature procedures. All reactions and manipulations were carried out under an atmosphere of prepurified nitrogen using either Schlenk techniques or an inertatmosphere glove box (Innovative Technologies). Ether solvents were distilled from $\mathrm{Na}$ /benzophenone prior to use. Hydrocarbon and chlorinated solvents were purified using a solvent purification system (Innovative Technologies; alumina / copper columns for hydrocarbon solvents), and the chlorinated solvents were subsequently distilled from $\mathrm{CaH}_{2}$ and degassed via several freeze-pump-thaw cycles.

All 499.893 MHz ${ }^{1} \mathrm{H}, 125.7 \mathrm{MHz}{ }^{13} \mathrm{C}, 160.4 \mathrm{MHz}{ }^{11} \mathrm{~B} \mathrm{NMR}$, and $99.25 \mathrm{MHz}{ }^{29} \mathrm{Si} \mathrm{NMR}$ spectra were recorded on a Varian INOVA spectrometer equipped with a boron-free $5 \mathrm{~mm}$ dual broadband gradient probe (Nalorac, Varian Inc., Martinez, CA). Solution ${ }^{1} \mathrm{H}$ and ${ }^{13} \mathrm{C}$ NMR spectra were referenced internally to solvent signals. ${ }^{11} \mathrm{~B}$ NMR spectra were acquired with boron-free quartz NMR tubes and the spectra were referenced externally to $\mathrm{BF}_{3} \cdot \mathrm{Et}_{2} \mathrm{O}(\square=0) .{ }^{29} \mathrm{Si} \mathrm{NMR}$ spectra were referenced to $\mathrm{SiMe}_{4}(\square=0)$. All NMR spectra were recorded at ambient temperature unless noted otherwise. The abbreviations Th (2-thienyl), $\mathrm{Hx}_{2} \mathrm{Th}_{2}$ (3,3'-dihexyl-2,2'-bithiophenediyl), $\mathrm{Ph}$ (phenyl), Mes (2,4,6-trimethylphenyl), and Hx (n-hexyl) are used for the peak assignments.

\section{Labeling Scheme:}

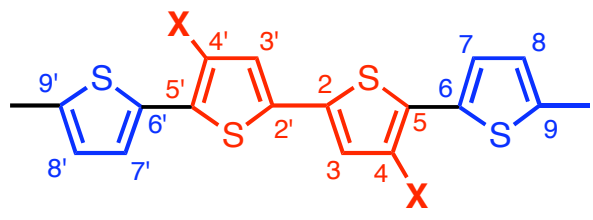

QT-X

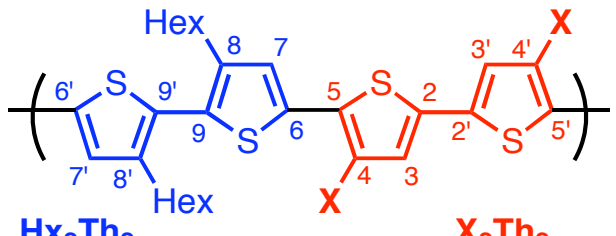

PT-X 
UV-visible absorption data were acquired on a Varian Cary 500 UV-Vis / NIR spectrophotometer. The fluorescence data and quantum yields were measured on a Varian Cary Eclipse Fluorescence spectrophotometer with the same solutions as those used in the UV-visible measurements. Anthracene was used as the standard for determination of the quantum yields $(\square)$. The quantum yield of anthracene was adopted from the 'Handbook of Photochemistry, $2^{\text {nd }}$ Edition, Revised and Expanded. ISBN 0-8247-7911-8, ${ }^{4}$ and the concentration of anthracene in $\mathrm{CH}_{2} \mathrm{Cl}_{2}$ was $1.12 \square 10^{-5} \mathrm{M}$. Sample solutions were prepared using a microbalance $( \pm 0.1 \mathrm{mg})$ and volumetric glassware.

GPC analyses were performed in THF ( $1 \mathrm{ml} / \mathrm{min})$ using a Waters Breeze system equipped with a 717plus autosampler, a 1525 binary HPLC pump, a 2487 dual $\square$ absorbance detector, and a 2414 refractive index detector. For separation the samples were passed through a series of styragel columns (Polymer Laboratories; two columns $5 \mathrm{Dm} / \mathrm{mixed}-\mathrm{C}$ ), which were kept in a column heater at $35^{\circ} \mathrm{C}$. The columns were calibrated with polystyrene standards (Polymer Laboratories). Multi-angle laser light scattering (MALLS) experiments were performed at $690 \mathrm{~nm}(30 \mathrm{~mW}$ linear polarized GaAs laser) using a Wyatt Dawn EOS instrument in-line with the GPC; differential refractive indices $\mathrm{d} n / \mathrm{d} c$ were calculated from in-line GPC-MALLS mode with the Wyatt Astra software assuming $100 \%$ mass recovery.

GC-MS spectra were acquired on a Hewlett Packard HP 6890 Series GC system equipped with a series 5973 mass selective detector and a series 7683 injector. A temperature profile with a heating rate of $20{ }^{\circ} \mathrm{C} / \mathrm{min}$ from $50{ }^{\circ} \mathrm{C}$ to $300{ }^{\circ} \mathrm{C}$ was used. MALDI-TOF-TOF measurements were performed on an Applied Biosystems 4700 Proteomics Analyzer as specified either in linear or in reflectron (+)mode with delayed extraction. Benzo[a]pyrene was used as the matrix $(10 \mathrm{mg} / \mathrm{mL}$ toluene). Samples were prepared in toluene $(10 \mathrm{mg} / \mathrm{mL})$, mixed with the matrix in a 1:10 ratio, and then spotted on the wells of a sample plate inside a glove box. Peptides were used for calibration (Des-Arg-Bradykinin (904.4681), Angiotensin I (1296.6853), Glu-Fibrinopeptide B (1570.6774), ACTH (clip 1-17) (2093.0867), ACTH (clip 18-39) (2465.1989), ACTH (clip 7-38) (3657.9294) with $\square$-hydroxy-4-cyanocinnamic acid as the matrix).

Cyclic voltammetry was carried out on a CV-50W analyzer from BAS. The three-electrode system consisted of an Au disk as working electrode, a Pt wire as secondary electrode and a Ag wire as the reference electrode. The voltammograms were recorded with ca. $10^{-3}$ to $10^{-4} \mathrm{M}$ solution in THF (reduction waves) or $\mathrm{CH}_{2} \mathrm{Cl}_{2}$ (oxidation waves) containing $\mathrm{Bu}_{4} \mathrm{~N}\left[\mathrm{PF}_{6}\right](0.1 \mathrm{M})$ as the supporting electrolyte. The scans were referenced after the addition of a small amount of ferrocene (reduction cycles) or decamethylferrocene (oxidation cycles) as internal standard. The potentials are reported relative to the ferrocene/ferrocenium couple.

Elemental analyses were obtained from Quantitative Technologies Inc. Whitehouse, NJ.

Differential scanning calorimetry (DSC) measurements were performed on a Perkin Elmer Differential Scanning Calorimeter Pyris 1 system with ca. $10 \mathrm{mg}$ of polymer and at a scan rate of $20{ }^{\circ} \mathrm{C} / \mathrm{min}$. The results reported are from the second heating cycle.

X-ray diffraction intensities were collected on a Bruker SMART APEX CCD diffractometer. Crystallographic data for QT-SiMe $\mathbf{B}_{3}$ and $\mathbf{Q T}-\mathbf{B M e s}_{2}$ and details of X-ray diffraction experiments and crystal structure refinements are given in Tables $\mathrm{S} 1$ and $\mathrm{S} 2$. SADABS ${ }^{5}$ absorption corrections were applied in both cases. Structures were solved using direct methods and completed by subsequent difference Fourier syntheses and refined by full matrix least-squares procedures on

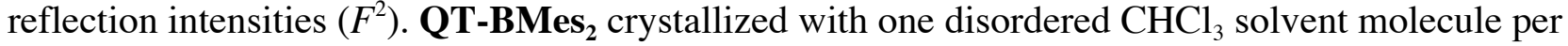
main molecule. The $\mathrm{CHCl}_{3}$ moieties were found with the $\mathrm{C}, \mathrm{H}$, and one of the $\mathrm{Cl}$ atoms at 95.8/4.2 
ratio, another $\mathrm{Cl}$ at 93.4/6.6, and the third $\mathrm{Cl}$ at 92.2/7.8. All non-hydrogen atoms were refined with anisotropic displacement coefficients. The $\mathrm{H}$ atoms were placed at calculated positions and were refined as riding atoms. All software and sources scattering factors are contained in the SHELXTL (5.10) program package ${ }^{6}$. Crystallographic data for the structures of QT-SiMe 3 and QT-BMes $_{2}$ have been deposited with the Cambridge Crystallographic Data Center as supplementary publications CCDC-641218 and CCDC-641219, respectively. Copies of the data can be obtained free of charge on application to CCDC, 12 Union Road, Cambridge CB2 1EZ, UK (fax: (+44) 1223-336-033; email: deposit@ccdc.cam.ac.uk).

DFT calculations have been performed with the Gaussian03 program. ${ }^{7,8}$ Geometries and electronic properties are calculated by means of hybrid density functional B3LYP with the basis set of $6-31 \mathrm{G}(\mathrm{d})$. The input files and orbital representations were generated with Gaussview (scaling radii of 75\%, isovalue of 0.02). Excitation data were calculated using TD-DFT (B3LYP).

Caution! $\mathrm{BBr}_{3}$ is toxic and highly corrosive and should be handled appropriately with great care. Fluorinated grease was used for ground glass joints in reactions involving $\mathrm{BBr}_{3}$.

\section{Synthesis of Borylated Polythiophenes}

Synthesis of 5,5'-Diiodo-4,4'-bis(trimethylsilyl)-2,2'-bithiophene $\left(\mathbf{T h}_{2} \mathbf{I}_{2}\left(\mathbf{S i M e}_{3}\right)_{2}\right)$ : 4,4'-Bis(trimethylsilyl)-2,2'-bithiophene $(20 \mathrm{~g}, 0.064 \mathrm{~mol})$ was dissolved in $500 \mathrm{ml}$ of dry THF with 'BuOK (1.0 g, $0.013 \mathrm{~mol})$. The solution was cooled to $-78{ }^{\circ} \mathrm{C}$ and ${ }^{\mathrm{t}} \mathrm{BuLi}(90.2 \mathrm{ml}, 1.5 \mathrm{M}, 0.134$ mol) was added over $1 \mathrm{~h}$. The reaction mixture was kept stirring for $1 \mathrm{~h}$ at this temperature and then stirred at room temp for $3 \mathrm{~h}$. The reaction mixture was cooled back to $-78^{\circ} \mathrm{C}$, and a solution of $\mathrm{I}_{2}(32.7 \mathrm{~g}, 0.128 \mathrm{~mol})$ in $100 \mathrm{ml}$ THF was added. The reaction mixture was allowed to warm up to room temperature and kept stirring overnight. After standard workup the crude brown material was purified by recrystallization from ethanol/ether to obtain colorless crystals ( $26 \mathrm{~g}, 72 \%)$. For $\mathrm{Th}_{2} \mathrm{I}_{2}\left(\mathrm{SiMe}_{3}\right)_{2}:{ }^{1} \mathrm{H}$ NMR $\left(\mathrm{CDCl}_{3}, 499.893 \mathrm{MHz}\right): \mathrm{C}=6.82\left(\mathrm{~s}, 2 \mathrm{H}, \mathrm{Th}_{2}-\mathrm{H} 4,4\right.$ '), $0.27\left(\mathrm{~s}, \mathrm{CH}_{3}\right) ;{ }^{13} \mathrm{C}$ NMR $\left(\mathrm{CDCl}_{3}, 125.7 \mathrm{MHz}\right): \mathrm{Z}=148.6,142.6,131.7,81.2(\mathrm{Th}),-0.4\left(\mathrm{Si}_{-} \mathrm{CH}_{3}\right) ;{ }^{29} \mathrm{Si} \mathrm{NMR}\left(\mathrm{CDCl}_{3}\right.$, 99.25 MHz): $\square=-5.1$; GC-MS m/z (\%): $562\left[\mathrm{M}^{+}\right](100), 547\left[\mathrm{M}^{+}-\mathrm{CH}_{3}\right]$ (11). Elemental analysis calcd for $\mathrm{C}_{14} \mathrm{H}_{20} \mathrm{I}_{2} \mathrm{~S}_{2} \mathrm{Si}_{2}: \mathrm{C} 29.90, \mathrm{H} 3.58$; found C $30.08, \mathrm{H} 3.53 \%$.

Synthesis of Silyl-functionalized Polymer PT-SiMe ${ }_{3} \cdot \mathrm{Pd}_{2}(\mathrm{dba})_{3}(0.32 \mathrm{~g}, 1 \mathrm{~mol} \%)$ and ${ }^{\mathrm{t}} \mathrm{Bu}_{3} \mathrm{P}(0.50$ g, $8 \mathrm{~mol} \%$ ) were dissolved in $10 \mathrm{ml}$ of $1: 1 \mathrm{THF} / \mathrm{DMF}$ mixture and kept stirring for $0.5 \mathrm{~h}$. $\mathrm{Th}_{2} \mathrm{Hx}_{2}\left(\mathrm{SnMe}_{3}\right)_{2}(10.0 \mathrm{~g}, 15.0 \mathrm{mmol})$ in $10 \mathrm{ml}$ of 1:1 THF/DMF mixture and $\mathrm{Th}_{2} \mathrm{I}_{2}\left(\mathrm{SiMe}_{3}\right)_{2}(8.38$ $\mathrm{g}, 14.9 \mathrm{mmol}$ ) in $10 \mathrm{ml}$ of 1:1 THF/DMF mixture were added slowly under stirring. The reaction mixture was heated to $80^{\circ} \mathrm{C}$ and kept stirring for 3 days. The completion of the reaction was apparent from precipitation of the catalyst. The reaction mixture was filtered through a frit and concentrated to $2 \mathrm{ml}$ under high vacuum. The polymer was purified by repeated precipitation (twice from THF into methanol and twice from THF into acetone). The yellow colored product was dried overnight at $60{ }^{\circ} \mathrm{C}$ under high vacuum. Yield: $6.40 \mathrm{~g}(66 \%)$. For PT-SiMe ${ }_{3}{ }^{1} \mathrm{H}$ NMR $\left(\mathrm{CDCl}_{3}, 499.893 \mathrm{MHz}\right): \square=7.15$ (s, $\left.2 \mathrm{H}, \mathrm{Th}-\mathrm{H}\right), 7.02(\mathrm{~s}, 2 \mathrm{H}, \mathrm{Th}-\mathrm{H}), 2.60\left(\mathrm{t},{ }^{3} \mathrm{~J}=7.5 \mathrm{~Hz}, 4 \mathrm{H}, \mathrm{Th}-\right.$ $\left.\mathrm{CH}_{2}\right), 1.60\left(\mathrm{~m}, 4 \mathrm{H}\right.$, hexyl), 1.35-1.25 (m, $12 \mathrm{H}$, hexyl), $0.89\left(\mathrm{t},{ }^{3} \mathrm{~J}=7.5 \mathrm{~Hz}, 6 \mathrm{H}, \mathrm{CH}_{3}\right), 0.29(\mathrm{~s}, 18 \mathrm{H}$, $\left.\mathrm{Si}-\mathrm{CH}_{3}\right) ; \mathrm{Hx}_{2} \mathrm{Th}_{2}-\mathrm{H}$ end groups: $7.32(\mathrm{~d}), 7.14$ (s), 6.99 (d), $2.52(\mathrm{t}) ; \mathrm{Si}_{2} \mathrm{Th}_{2}-\mathrm{Me}$ groups: 6.98 (s), $2.55(\mathrm{~s}) ;{ }^{13} \mathrm{C} \mathrm{NMR}\left(\mathrm{CDCl}_{3}, 125.7 \mathrm{MHz}\right): \square=143.0,141.5,141.1,137.0,136.9,131.0,129.9,129.6$ (Th), 31.9, 31.0, 29.3, 29.2, 22.9, 14.3 (hexyl), $0.5\left(\mathrm{Si}_{-} \mathrm{CH}_{3}\right) ;{ }^{29} \mathrm{Si} \mathrm{NMR}\left(\mathrm{CDCl}_{3}, 99.25 \mathrm{MHz}\right): \square=$ -7.9; GPC-RI (in THF against PS standards) $M_{\mathrm{w}}=14033, P D I=1.53$; GPC-MALLS (THF, $\mathrm{d} n / \mathrm{d} c$ 
$=0.223 \mathrm{~mL} / \mathrm{g}) M_{\mathrm{w}}=9107, P D I=1.35 ; \mathrm{UV}-\mathrm{Vis}\left(\mathrm{CH}_{2} \mathrm{Cl}_{2}, 6.17 \square 10^{-5} \mathrm{M}\right): \square_{\max }=382 \mathrm{~nm}(\square=$ $15800)$; fluorescence $\left(\mathrm{CH}_{2} \mathrm{Cl}_{2}, 6.17 \square 10^{-5} \mathrm{M}\right): \square_{\mathrm{em} \text {,max }}=554 \mathrm{~nm}\left(\square_{\mathrm{exc}}=380 \mathrm{~nm}\right) ; \square=10 \% ; \mathrm{CV}$ $\left(\mathrm{CH}_{2} \mathrm{Cl}_{2} / 0.1 \mathrm{M} \mathrm{Bu}_{4} \mathrm{~N}\left[\mathrm{PF}_{6}\right], 100 \mathrm{mV} / \mathrm{s}\right.$, vs. Fc/Fc ${ }^{+}$couple $): E_{1 / 2}(1)=0.45 \mathrm{~V}\left(\square E_{\mathrm{p}}=74 \mathrm{mV}\right)$ and $E_{1 / 2}(2)=0.70 \mathrm{~V}\left(\square E_{\mathrm{p}}=84 \mathrm{mV}\right) ; \mathrm{CV}\left(\mathrm{THF} / 0.1 \mathrm{M} \mathrm{Bu}_{4} \mathrm{~N}\left[\mathrm{PF}_{6}\right], 100 \mathrm{mV} / \mathrm{s}\right.$, vs. Fc/Fc ${ }^{+}$couple $): E_{1 / 2}(1)$ $=-2.35 \mathrm{~V}\left(\square E_{\mathrm{p}}=157 \mathrm{mV}\right)$ and $E_{\mathrm{p}, \mathrm{a}}(2)=-2.54 \mathrm{~V}\left(\left(\square E_{\mathrm{p}}=195 \mathrm{mV}\right) ; \mathrm{DSC}\left(20{ }^{\circ} \mathrm{C} / \mathrm{min}\right): T_{\mathrm{g}}=58\right.$ ${ }^{\circ} \mathrm{C}$ ); TGA $\left(\mathrm{N}_{2}, 20{ }^{\circ} \mathrm{C} / \mathrm{min}\right.$ ): $T_{\mathrm{dec}}=350{ }^{\circ} \mathrm{C}$ (onset, $92 \%$ weight loss at $900{ }^{\circ} \mathrm{C}$ ); elemental analysis calcd for $\left(\mathrm{C}_{17} \mathrm{H}_{24} \mathrm{~S}_{2} \mathrm{Si}\right)_{\mathrm{n}}$ : C 63.69, $\mathrm{H} 7.55$; found C 62.40, H 7.18\%.

Reaction of PT-SiMe ${ }_{3}$ with $\mathbf{B B r}_{3}$ : Synthesis of PT-BBr ${ }_{2}$. A solution of PT-SiMe $320 \mathrm{mg}, 1.0$ mmol) in $10 \mathrm{ml}$ of $\mathrm{CH}_{2} \mathrm{Cl}_{2}$ was added dropwise to a solution of $\mathrm{BBr}_{3}(324 \mathrm{mg}, 1.3 \mathrm{mmol})$ in $5 \mathrm{ml}$ $\mathrm{CH}_{2} \mathrm{Cl}_{2}$ under stirring. The reaction mixture was stirred for $24 \mathrm{~h}$, and the resulting polymer solution was used directly for further reactions with nucleophiles. For PT-BBr $2:{ }^{1} \mathrm{H} \mathrm{NMR}\left(\mathrm{CDCl}_{3}\right.$, $499.893 \mathrm{MHz}$ ): $\square=7.66$ (br, 2H, B $\mathrm{Th}_{2}-\mathrm{H} 3,3$ '), 7.12 (br, 2H, $\mathrm{Hx}_{2} \mathrm{Th}_{2}-\mathrm{H} 7,7$ '), 2.61 (br, 4H, Th$\mathrm{CH}_{2}$ ), 1.60 (br, 4H, hexyl), 1.35-1.25 (m, 12H, hexyl), 0.90 (br, $6 \mathrm{H}$, hexyl- $\mathrm{CH}_{3}$ ); $\mathrm{Hx}_{2} \mathrm{Th}_{2}-\mathrm{H}$ end groups: 7.33 (d), 7.00 (d); $\mathrm{Hx}_{2} \mathrm{Th}_{2}-\mathrm{BMes}_{2}$ end groups: $7.92(\mathrm{~s}) ; \mathrm{B}_{2} \mathrm{Th}_{2}-\mathrm{Me}$ groups: $7.58(\mathrm{~s}), 2.87$ (s, $\left.\mathrm{CH}_{3}\right) ;{ }^{13} \mathrm{C} \mathrm{NMR}\left(\mathrm{CDCl}_{3}, 125.7 \mathrm{MHz}\right): \square=150.5,143.9,142.1,134.8,134.1,132.7,132.2,131.5$ (Th), 31.8, 30.9, 29.3, 22.8, 14.3 (hexyl); ${ }^{11} \mathrm{~B}$ NMR $\left(\mathrm{CDCl}_{3}, 160.3 \mathrm{MHz}\right): \square=50.1\left(w_{1 / 2}=2100\right.$ $\mathrm{Hz})$.

Synthesis of PT-BMes ${ }_{2}$. A solution of PT-BBr 2 prepared from $\mathrm{BBr}_{3}(324 \mathrm{mg}, 1.3 \mathrm{mmol})$ and PT$\mathrm{SiMe}_{3}(320 \mathrm{mg} ; 1.0 \mathrm{mmol})$ in $15 \mathrm{ml}$ of toluene was treated with "MesCu" (600 mg $\left.2.8 \mathrm{mmol}\right)$ in 5 $\mathrm{ml}$ of toluene. The reaction mixture was then heated to $100{ }^{\circ} \mathrm{C}$ for $24 \mathrm{~h}$. A solid precipitate $(\mathrm{CuBr})$ formed and was removed by filtration through a fritted glass disk. The reaction solution was concentrated under high vacuum to $2 \mathrm{ml}$ and then precipitated into $500 \mathrm{ml}$ of cold hexanes (ice bath). The red powdery material was washed with cold hexanes and dried under high vacuum for 12 h. Yield: $0.34 \mathrm{~g}$ (68 \%). For PT-BMes 2 : ${ }^{1} \mathrm{H}$ NMR $\left(\mathrm{CDCl}_{3}, 499.893 \mathrm{MHz}\right): \square=6.94$ (br, $2 \mathrm{H}$, $\mathrm{B}_{2} \mathrm{Th}_{2}-\mathrm{H} 3,3^{\prime}$ ), 6.69 (br, 8H, Mes-H), 6.48 (br, 2H, Hx ${ }_{2} \mathrm{Th}_{2}-\mathrm{H} 7,7^{\prime}$ ), 2.23 (s, 12H, Mes ${ }_{p}-\mathrm{CH}_{3}$ ), 2.08 (s, 28H, $\mathrm{Mes}_{o}-\mathrm{CH}_{3}$ and Th- $\left.\mathrm{CH}_{2}\right), 1.3-1.2\left(\mathrm{~m}, 16 \mathrm{H}\right.$, hexyl), $0.89\left(\mathrm{t},{ }^{3} \mathrm{~J}=7.5 \mathrm{~Hz}, 6 \mathrm{H}\right.$, hexyl- $\left.\mathrm{CH}_{3}\right) ;{ }^{13} \mathrm{C}$ NMR $\left(\mathrm{CDCl}_{3}, 125.7 \mathrm{MHz}\right): \square=148.9$ (Th), 145.4 (Th), 142.4 (Th), 142.2 (Mes), 140.9 (Mes),

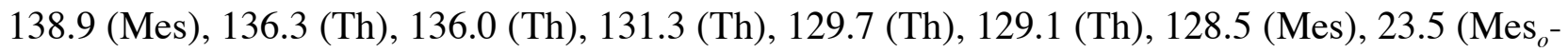
$\left.\mathrm{CH}_{3}\right), 21.5\left(\mathrm{Mes}_{p}-\mathrm{CH}_{3}\right), 31.9,30.4,29.9,29.3,22.9,14.4$ (hexyl); ${ }^{11} \mathrm{~B} \mathrm{NMR}\left(\mathrm{CDCl}_{3}, 160.3 \mathrm{MHz}\right)$ : $\square=62\left(w_{1 / 2}=5100 \mathrm{~Hz}\right)$; GPC-RI (in THF against PS standards) $M_{\mathrm{w}}=15036, P D I=1.63$; GPCMALLS (THF, $\mathrm{d} n / \mathrm{d} c=0.216 \mathrm{~mL} / \mathrm{g}) M_{\mathrm{w}}=20650, P D I=1.88 ; \mathrm{UV}-\mathrm{Vis}\left(\mathrm{CH}_{2} \mathrm{Cl}_{2}, 8.28 \square 10^{-5} \mathrm{M}\right)$ : $\square_{\max }=344 \mathrm{~nm}(\square=18100), 441 \mathrm{~nm}(\square=10600)$; fluorescence $\left(\mathrm{CH}_{2} \mathrm{Cl}_{2}, 8.28 \square 10^{-5} \mathrm{M}\right): \square_{\mathrm{em}, \max }=$ $617 \mathrm{~nm}\left(\square_{\mathrm{exc}}=440 \mathrm{~nm}\right) ; \square=3.3 \%$; fluorescence (thin film): $\square_{\mathrm{em}, \max }=617 \mathrm{~nm} ; \mathrm{CV}(\mathrm{THF} / 0.1 \mathrm{M}$ $\mathrm{Bu}_{4} \mathrm{~N}\left[\mathrm{PF}_{6}\right], 100 \mathrm{mV} / \mathrm{s}$, vs. $\mathrm{Fc} / \mathrm{Fc}^{+}$couple $) E_{1 / 2}(1)=-2.18 \mathrm{~V}\left(\square E_{\mathrm{p}}=96 \mathrm{mV}\right)$ and $E_{1 / 2}(2)=-2.41 \mathrm{~V}$ $\left(\square E_{\mathrm{p}}=95 \mathrm{mV}\right) ; \mathrm{CV}\left(\mathrm{CH}_{2} \mathrm{Cl}_{2} / 0.1 \mathrm{M} \mathrm{Bu}_{4} \mathrm{~N}\left[\mathrm{PF}_{6}\right], 100 \mathrm{mV} / \mathrm{s}\right.$, vs. Fc/Fc ${ }^{+}$couple $): E_{1 / 2}(1)=0.47 \mathrm{~V}$ $\left(\square E_{\mathrm{p}}=174 \mathrm{mV}\right)$ and $E_{\mathrm{p}}(2)=0.53 \mathrm{~V}$ (irreversible); DSC $\left(20^{\circ} \mathrm{C} / \mathrm{min}\right): T_{\mathrm{g}}$ (onset) $\left.=88.6^{\circ} \mathrm{C}\right)$; TGA $\left(\mathrm{N}_{2}, 20{ }^{\circ} \mathrm{C} / \mathrm{min}\right.$ ): $T_{\mathrm{dec}}=250{ }^{\circ} \mathrm{C}$ (onset, $80 \%$ weight loss at $750{ }^{\circ} \mathrm{C}$ ); elemental analysis calcd for $\left(\mathrm{C}_{32} \mathrm{H}_{37} \mathrm{~B}_{1} \mathrm{~S}_{2}\right)_{\mathrm{n}}$ : C 77.40, H 7.51; found C 74.93, H 7.08\%.

Solvent Dependence of Absorption/Emission Spectra for PT-BMes ${ }_{2}$ :

\begin{tabular}{lcccc}
\hline PT-BMes & \multicolumn{2}{c}{$D_{\text {abs, max }}$} & $\square_{\mathrm{em}, \max }$ & $\square$ \\
\hline Cyclohexane & 341 & 437 & 612 & 1.9 \\
$\mathrm{CH}_{2} \mathrm{Cl}_{2}$ & 344 & 441 & 617 & 3.3 \\
Pyridine & 346 & 442 & 615 & N/A \\
\hline
\end{tabular}




\section{Synthesis of Borylated Quaterthiophene Model Compounds:}

Synthesis of QT-SiMe $: \mathrm{Pd}_{2}(\mathrm{dba})_{3}(0.077 \mathrm{~g}, 1 \mathrm{~mol} \%)$ and ${ }^{\mathrm{t}} \mathrm{Bu}_{3} \mathrm{P}(0.12 \mathrm{~g}, 8 \mathrm{~mol} \%)$ were dissolved in $10 \mathrm{ml}$ of 1:1 THF/DMF and kept stirring for $0.5 \mathrm{~h}$. 2-Trimethylstannyl-5-methylthiophene (1.95 $\mathrm{g}, 7.46 \mathrm{mmol})$ in $5 \mathrm{ml}$ of THF followed by $\mathrm{Th}_{2} \mathrm{I}_{2}\left(\mathrm{SiMe}_{3}\right)_{2}(2.0 \mathrm{~g}, 3.5 \mathrm{mmol})$ in $10 \mathrm{ml}$ of THF were added slowly under stirring. The reaction mixture was heated to $80^{\circ} \mathrm{C}$ and kept stirring for $3 \mathrm{~d}$. The completion of the reaction was evident from precipitation of the catalyst. The reaction mixture was filtered through a frit and concentrated, and all volatile material was removed under high vacuum at $60{ }^{\circ} \mathrm{C}$. The crude product was recrystallized from toluene to obtain a yellow crystalline product $(1.22 \mathrm{~g}, 68 \%)$. For QT-SiMe $3:{ }^{1} \mathrm{H}$ NMR $\left(\mathrm{CDCl}_{3}, 499.893 \mathrm{MHz}\right): \square=7.09\left(\mathrm{~s}, 2 \mathrm{H}, \mathrm{Th}_{2}-\right.$ $\left.\mathrm{H} 3,3^{\prime}\right), 6.90\left(\mathrm{~d},{ }^{3} \mathrm{~J}=3.5 \mathrm{~Hz}, 2 \mathrm{H}, \mathrm{Th}_{2}-\mathrm{H} 8,8^{\prime}\right), 6.70\left(\mathrm{~m}, 2 \mathrm{H}, \mathrm{Th}_{2}-\mathrm{H} 7,7^{\prime}\right), 2.51\left(\mathrm{~s}, 6 \mathrm{H}, \mathrm{Th}^{-} \mathrm{CH}_{3}\right), 0.23$ (s, $\left.18 \mathrm{H}, \mathrm{Si}_{-} \mathrm{CH}_{3}\right) ;{ }^{13} \mathrm{C} \mathrm{NMR}\left(\mathrm{CDCl}_{3}, 125.7 \mathrm{MHz}\right): \square=142.1,141.3,140.6,136.8,134.7,130.7$, 128.2, $125.6(\mathrm{Th}), 15.6\left(\mathrm{Th}-\mathrm{CH}_{3}\right), 0.4\left(\mathrm{Si}_{-} \mathrm{CH}_{3}\right) ;{ }^{29} \mathrm{Si} \mathrm{NMR}\left(\mathrm{CDCl}_{3}, 99.25 \mathrm{MHz}\right): \square=-7.5$; GC-MS $\mathrm{m} / \mathrm{z}(\%): 502\left[\mathrm{M}^{+}\right](100), 487\left[\mathrm{M}^{+}-\mathrm{CH}_{3}\right](5)$; UV-Vis $\left(\mathrm{CH}_{2} \mathrm{Cl}_{2}, 3.8 \square 10^{-5} \mathrm{M}\right): \square_{\max }=348 \mathrm{~nm}(\square=$ $25280), 357 \mathrm{~nm}(\square=25160)$; fluorescence $\left(\mathrm{CH}_{2} \mathrm{Cl}_{2}, 3.8 \square 10^{-5} \mathrm{M}\right)$ : $\square_{\text {em,max }}=470,495 \mathrm{~nm}(\mathrm{sh})\left(\square_{\text {exc }}\right.$ $=355 \mathrm{~nm}) ; \mathrm{\square}=24 \% ; \mathrm{CV}\left(\mathrm{THF} / 0.1 \mathrm{M} \mathrm{Bu}_{4} \mathrm{~N}\left[\mathrm{PF}_{6}\right], 100 \mathrm{mV} / \mathrm{s}\right.$, vs. Fc/Fc ${ }^{+}$couple): $E_{1 / 2}(1)=-2.53 \mathrm{~V}$ $\left(\square E_{\mathrm{p}}=221 \mathrm{mV}\right)$ and $E_{1 / 2}(2)=-2.76 \mathrm{~V}\left(\square E_{\mathrm{p}}=276 \mathrm{mV}\right) ; \mathrm{CV}\left(\mathrm{CH}_{2} \mathrm{Cl}_{2} / 0.1 \mathrm{M} \mathrm{Bu}_{4} \mathrm{~N}\left[\mathrm{PF}_{6}\right], 100 \mathrm{mV} / \mathrm{s}\right.$, vs. Fc $/ \mathrm{Fc}^{+}$couple): $E_{1 / 2}(1)=0.48 \mathrm{~V}\left(\square E_{\mathrm{p}}=90 \mathrm{mV}\right)$ and $E_{1 / 2}(2)=0.72 \mathrm{~V}\left(\square E_{\mathrm{p}}=102 \mathrm{mV}\right)$; DSC (20 ${ }^{\circ} \mathrm{C} / \mathrm{min}$ ): $T_{\mathrm{m}}=153{ }^{\circ} \mathrm{C}$; MALDI-TOF-TOF $\mathrm{m} / \mathrm{z}(\%): 502.0881\left[\mathrm{M}^{+}\right]$; elemental analysis calcd for $\mathrm{C}_{24} \mathrm{H}_{30} \mathrm{~S}_{4} \mathrm{Si}_{2}$ : C 57.32, H 6.00, S 25.50; found C 57.16, H 6.01, S 25.62\%.

Reaction of QT-SiMe ${ }_{3}$ with $\mathbf{B B r}_{3}$ : Synthesis of QT-BBr ${ }_{2}$. A solution of QT-SiMe $\mathbf{S}_{3}(1.00 \mathrm{~g}, 2.00$ $\mathrm{mmol})$ in $10 \mathrm{ml}$ of $\mathrm{CH}_{2} \mathrm{Cl}_{2}$ was added dropwise under stirring to $\mathrm{BBr}_{3}(1.20 \mathrm{~g}, 4.73 \mathrm{mmol})$ in $5 \mathrm{ml}$ $\mathrm{CH}_{2} \mathrm{Cl}_{2}$. The reaction mixture was stirred for $6 \mathrm{~h}$. All volatile components were removed under high vacuum and the crude product was crystallized from toluene at $-35^{\circ} \mathrm{C}$. Yield: $1.02 \mathrm{~g}(72 \%)$. For QT-BBr ${ }_{2}:{ }^{1} \mathrm{H}$ NMR $\left(\mathrm{CDCl}_{3}, 499.893 \mathrm{MHz}\right): \square=7.59\left(\mathrm{~s}, 2 \mathrm{H}, \mathrm{Th}_{2}-\mathrm{H} 3,3^{\prime}\right), 7.01\left(\mathrm{~d},{ }^{3} \mathrm{~J}=3.5 \mathrm{~Hz}\right.$, $\left.2 \mathrm{H}, \mathrm{Th}_{2}-\mathrm{H} 8,8^{\prime}\right), 6.76\left(\mathrm{~m},{ }^{3} \mathrm{~J}=3.5 \mathrm{~Hz}, 2 \mathrm{H}, \mathrm{Th}_{2}-\mathrm{H} 7,7^{\prime}\right), 2.54\left(\mathrm{~s}, 6 \mathrm{H}, \mathrm{CH}_{3}\right) ;{ }^{13} \mathrm{C} \mathrm{NMR}\left(\mathrm{CDCl}_{3}, 125.7\right.$ $\mathrm{MHz}): \square=151.4,144.0,142.0$ (br) $134.5,132.6,131.7,130.6,126.4$ (Th), $15.7\left(\mathrm{Th}_{-} \mathrm{CH}_{3}\right) ;{ }^{11} \mathrm{~B}$ $\operatorname{NMR}\left(\mathrm{CDCl}_{3}, 160.3 \mathrm{MHz}\right): \square=50.7\left(w_{1 / 2}=550 \mathrm{~Hz}\right)$.

Reaction of QT-BBr ${ }_{2}$ with 4 equivalent of "MesCu": Synthesis of QT-BMes ${ }_{2}$. QT-BBr 2 (50.7 $\mathrm{mg}, 0.073 \mathrm{mmol})$ in $5 \mathrm{ml}$ of toluene was treated with "MesCu" $(60.6 \mathrm{mg}, 0.29 \mathrm{mmol})$ in $5 \mathrm{ml}$ of toluene. The reaction mixture was then heated to $100{ }^{\circ} \mathrm{C}$ for $24 \mathrm{~h}$. A solid precipitate $(\mathrm{CuBr})$ was removed by filtration through a fritted glass disk. All volatile components were removed under high vacuum, the crude product was chromatographed on alumina with toluene as the eluent, and then recrystallized from toluene at $-35^{\circ} \mathrm{C}$. Yield: $38 \mathrm{mg}(61 \%)$. Single crystals were obtained by recrystallization from $\mathrm{CDCl}_{3}$. For QT-BMes $2{ }^{1} \mathrm{H}$ NMR $\left(\mathrm{CDCl}_{3}, 499.893 \mathrm{MHz}\right): \square=6.89(\mathrm{~s}, 2 \mathrm{H}$, $\left.\mathrm{Th}_{2}-\mathrm{H} 3,3^{\prime}\right), 6.68$ (s, 8H, Mes), 6.53 (d, $\left.{ }^{3} \mathrm{~J}=3.5 \mathrm{~Hz}, 2 \mathrm{H}, \mathrm{Th}_{2}-\mathrm{H} 8,8^{\prime}\right), 6.31$ (br d, ${ }^{3} \mathrm{~J}=3.5 \mathrm{~Hz}, 2 \mathrm{H}$, $\mathrm{Th}_{2}-\mathrm{H} 7,7$ '), 2.29 (s, 6H, Th- $\mathrm{CH}_{3}$ ), 2.24 (s, 12H, $\mathrm{Mes}_{p}-\mathrm{CH}_{3}$ ), 2.07 (s, 24H, $\left.\mathrm{Mes}_{0}-\mathrm{CH}_{3}\right) ;{ }^{13} \mathrm{C} \mathrm{NMR}$ $\left(\mathrm{CDCl}_{3}, 125.7 \mathrm{MHz}\right): \square=148.5$ (Th), $145.6(\mathrm{Th}), 142.4$ (Mes), 141.3 (Th), 141.0 (Mes), 138.9 (Mes), 135.8 (Th), 134.1 (Th), 131.1 (Th), 128.3 (Mes), 127.3 (Th), 125.4 (Th), $23.4\left(\mathrm{Mes}_{0}-\mathrm{CH}_{3}\right.$ ), $21.4\left(\mathrm{Mes}_{p}-\mathrm{CH}_{3}\right), 15.3\left(\mathrm{Th}_{-} \mathrm{CH}_{3}\right) ;{ }^{11} \mathrm{~B} \mathrm{NMR}\left(\mathrm{CDCl}_{3}, 160.3 \mathrm{MHz}\right): \square=69\left(w_{1 / 2}=2000 \mathrm{~Hz}\right) ; \mathrm{UV}-\mathrm{Vis}$ $\left(\mathrm{CH}_{2} \mathrm{Cl}_{2}, 5.45 \square 10^{-5} \mathrm{M}\right): \square_{\max }=275(\square=28000), 340(\square=40330), 412 \mathrm{~nm}(\square=16600)$, fluorescence $\left(\mathrm{CH}_{2} \mathrm{Cl}_{2}, 5.45 \square 10^{-5} \mathrm{M}\right): \square_{\mathrm{em}, \text { max }}=560 \mathrm{~nm}\left(\square_{\mathrm{exc}}=340 \mathrm{~nm}\right) ; \square=1.5 \% ; \mathrm{CV}(\mathrm{THF} /$ $0.1 \mathrm{M} \mathrm{Bu}_{4} \mathrm{~N}\left[\mathrm{PF}_{6}\right], 100 \mathrm{mV} / \mathrm{s}$, vs. Fc/Fc $/ \mathrm{Fc}^{+}$couple $): E_{1 / 2}(1)=-2.27 \mathrm{~V}\left(\square E_{\mathrm{p}}=108 \mathrm{mV}\right)$ and $E_{1 / 2}(2)=$ $-2.44 \mathrm{~V}\left(\square E_{\mathrm{p}}=158 \mathrm{mV}\right) ; \mathrm{CV}\left(\mathrm{CH}_{2} \mathrm{Cl}_{2} / 0.1 \mathrm{M} \mathrm{Bu}_{4} \mathrm{~N}\left[\mathrm{PF}_{6}\right], 100 \mathrm{mV} / \mathrm{s}\right.$, vs. $\mathrm{Fc} / \mathrm{Fc}^{+}$couple $): E_{1 / 2}(1)=$ 
$0.51 \mathrm{~V}\left(\square E_{\mathrm{p}}=96 \mathrm{mV}\right)$ and $E_{1 / 2}(2)=0.76 \mathrm{~V}\left(\square E_{\mathrm{p}}=156 \mathrm{mV}\right)$; High-resolution MALDI-TOF-TOF: $\mathrm{m} / \mathrm{z}=854.3375$ (calcd for ${ }^{12} \mathrm{C}_{54}{ }^{1} \mathrm{H}_{56}{ }^{11} \mathrm{~B}_{2}{ }^{32} \mathrm{~S}_{4} 854.3451$ ).

NMR reaction of $\mathbf{T h}_{2} \mathbf{H x}_{2}$ with $\mathbf{B B r}_{3}$. At room temperature, a solution of $\mathrm{Th}_{2} \mathrm{Hx}_{2}$ (18 mg, 0.054 mmol $)$ in $\mathrm{C}_{6} \mathrm{D}_{6}$ was added to solution of $\mathrm{BBr}_{3}(27 \mathrm{mg}, 0.108 \mathrm{mmol}) \mathrm{C}_{6} \mathrm{D}_{6}$. After $2 \mathrm{~h}$ the mixture was studied by ${ }^{1} \mathrm{H}$ NMR, which revealed two sets of signals in the aromatic region, one for the starting material $\left(\square=6.94\left(\mathrm{~d},{ }^{3} \mathrm{~J}=5.0 \mathrm{~Hz}, 2 \mathrm{H}\right), 6.79\left(\mathrm{~d},{ }^{3} \mathrm{~J}=5.0 \mathrm{~Hz}, 2 \mathrm{H}\right)\right)$ and a new set (ca. $25 \%$ intensity) for the mono-borylated product $\left(\square=7.86(\mathrm{~s}, 1 \mathrm{H}), 6.92\left(\mathrm{~d},{ }^{3} \mathrm{~J}=5.0 \mathrm{~Hz}, 1 \mathrm{H}\right), 6.72\left(\mathrm{~d},{ }^{3} \mathrm{~J}=\right.\right.$ $5 \mathrm{~Hz}, 1 \mathrm{H}$ ). With a larger excess of $\mathrm{BBr}_{3}$ (ca. 4 equiv), and after overnight reaction, one more set of signals was observed, which is attributed to the 5,5'-diborylated species $(\square 7.78(\mathrm{~s}, 2 \mathrm{H}))$.

\section{Comparison of GPC Results:}

\begin{tabular}{ccccccccc}
\hline Sample & \multicolumn{4}{c}{ GPC-RI } & \multicolumn{5}{c}{ GPC-LS } \\
& $\mathrm{M}_{\mathrm{w}}$ & $\mathrm{M}_{\mathrm{n}}$ & PDI & $\mathrm{DP}\left(\mathrm{M}_{\mathrm{n}}\right)$ & $\mathrm{M}_{\mathrm{w}}$ & $\mathrm{M}_{\mathrm{n}}$ & PDI & DP $\left(\mathrm{M}_{\mathrm{n}}\right)$ \\
PTh-SiMe $_{3}$ & 14033 & 9164 & 1.53 & 14.3 & 9107 & 6727 & 1.35 & 10.5 \\
PTh-BMes $_{2}$ & 15036 & 9229 & 1.63 & 9.3 & 20650 & 10990 & 1.88 & 11.1 \\
\hline
\end{tabular}

\section{References:}

(1) Lukevics, E.; Arsenyan, P.; Belyakov, S.; Popelis, J.; Pudova, O. Organometallics 2001, 20, 2487-2491.

(2) Seitz, D. E.; Lee, S. H.; Hanson, R. N.; Bottaro, J. C. Synth. Commun. 1983, 13, 121-128.

(3) (a) Tsuda, T.; Yazawa, T.; Watanabe, K.; Fujii, T.; Saegusa, T. J. Org. Chem. 1981, 46, 192-194. (b) Gambarotta, S.; Floriani, C.; Chiesi-Villa, A.; Guastini, C. J. Chem. Soc., Chem. Commun. 1983, 1156-1158. (c) Eriksson, H.; Håkansson, M. Organometallics 1997, 16, 4243-4244.

(4) Murov, S. L.; Carmichael, I.; Hug, G. L., Eds. Handbook of Photochemistry; 2nd ed.; Marcel Dekker Inc.: New York, 1993.

(5) Sheldrick, G.M. (1998), SADABS (2.01), Bruker/Siemens Area Detector Absorption Correction Program, Bruker AXS, Madison, Wisconsin, USA

(6) Sheldrick, G., Bruker XRD, Madison, WI.

(7) Gaussian 03, Revision C.02, M. J. Frisch, G. W. Trucks, H. B. Schlegel, G. E. Scuseria, M. A. Robb, J. R. Cheeseman, J. A. Montgomery, Jr., T. Vreven, K. N. Kudin, J. C. Burant, J. M. Millam, S. S. Iyengar, J. Tomasi, V. Barone, B. Mennucci, M. Cossi, G. Scalmani, N. Rega, G. A. Petersson, H. Nakatsuji, M. Hada, M. Ehara, K. Toyota, R. Fukuda, J. Hasegawa, M. Ishida, T. Nakajima, Y. Honda, O. Kitao, H. Nakai, M. Klene, X. Li, J. E. Knox, H. P. Hratchian, J. B. Cross, C. Adamo, J. Jaramillo, R. Gomperts, R. E. Stratmann, O. Yazyev, A. J. Austin, R. Cammi, C. Pomelli, J. W. Ochterski, P. Y. Ayala, K. Morokuma, G. A. Voth, P. Salvador, J. J. Dannenberg, V. G. Zakrzewski, S. Dapprich, A. D. Daniels, M. C. Strain, O. Farkas, D. K. Malick, A. D. Rabuck, K. Raghavachari, J. B. Foresman, J. V. Ortiz, Q. Cui, A. G. Baboul, S. Clifford, J. Cioslowski, B. B. Stefanov, G. Liu, A. Liashenko, P. Piskorz, I. Komaromi, R. L. Martin, D. J. Fox, T. Keith, M. A. AlLaham, C. Y. Peng, A. Nanayakkara, M. Challacombe, P. M. W. Gill, B. Johnson, W. Chen, M. W. Wong, C. Gonzalez, and J. A. Pople, Gaussian, Inc., Wallingford CT, 2004. 
(8) (a) Becke, A. D. J. Chem. Phys. 1993, 98, 5648-5652. (b) Tozer, D. T.; Handy, N. C. Phys. Chem. 2000, 2, 2117-2121; (c) Becke, A. D. J. Chem. Phys. 1996, 104, 1040-1046; (d) Casida, M. E.; Jamorski, C.; Casida, K. C.; Salahub, D. R. J. Chem. Phys. 1998, 108, 4439-4449 
Table S1. Selected Interatomic Distances [ $[\AA]$, and Angles [ $\left.{ }^{\circ}\right]$ for $\mathbf{Q T - S i M e}{ }_{3}$ and $\mathbf{Q T}-\mathbf{B M e s}_{2}$.

\begin{tabular}{|c|c|c|c|c|c|}
\hline \multicolumn{2}{|c|}{$\mathrm{QT}_{-\mathrm{SiMe}_{3}}$ - asymmetric } & \multicolumn{2}{|c|}{ 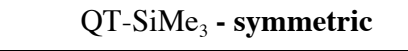 } & \multicolumn{2}{|c|}{ 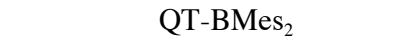 } \\
\hline $\mathrm{C} 1-\mathrm{C} 2, \mathrm{C} 17-\mathrm{C} 18$ & $1.500(2), 1.503(2)$ & $\mathrm{C} 25-\mathrm{C} 26$ & $1.504(2)$ & $\mathrm{C} 26-\mathrm{C} 27$ & $1.495(2)$ \\
\hline $\mathrm{C} 2-\mathrm{C} 3, \mathrm{C} 16-\mathrm{C} 17$ & $1.360(2), 1.358(2)$ & $\mathrm{C} 26-\mathrm{C} 27$ & $1.354(2)$ & $\mathrm{C} 26-\mathrm{C} 25$ & $1.358(3)$ \\
\hline $\mathrm{C} 3-\mathrm{C} 4, \mathrm{C} 15-\mathrm{C} 16$ & $1.420(2), 1.421(2)$ & $\mathrm{C} 27-\mathrm{C} 28$ & $1.421(2)$ & $\mathrm{C} 25-\mathrm{C} 24$ & $1.415(3)$ \\
\hline C4-C5, C14-C15 & $1.369(2), 1.371(2)$ & $\mathrm{C} 28-\mathrm{C} 29$ & $1.367(2)$ & $\mathrm{C} 24-\mathrm{C} 23$ & $1.371(3)$ \\
\hline $\mathrm{S} 1-\mathrm{C} 2, \mathrm{~S} 4-\mathrm{C} 17$ & $1.7335(15), 1.7337(15)$ & S5-C26 & $1.7321(16)$ & S2-C26 & $1.7264(18)$ \\
\hline S1-C5, S4-C14 & $1.7335(15), 1.7361(15)$ & S5-C29 & $1.7372(15)$ & $\mathrm{S} 2-\mathrm{C} 23$ & $1.7365(17)$ \\
\hline C6-C7, C12-C13 & $1.381(2), 1.383(2)$ & C30-C31 & $1.384(4)$ & $\mathrm{C} 22-\mathrm{C} 21$ & $1.384(4)$ \\
\hline $\mathrm{C} 7-\mathrm{C} 8, \mathrm{C} 11-\mathrm{C} 12$ & $1.431(2), 1.428(2)$ & $\mathrm{C} 31-\mathrm{C} 32$ & $1.431(2)$ & $\mathrm{C} 20-\mathrm{C} 21$ & $1.433(2)$ \\
\hline $\mathrm{C} 8-\mathrm{C} 9, \mathrm{C} 10-\mathrm{C} 11$ & $1.369(2), 1.369(2)$ & $\mathrm{C} 32-\mathrm{C} 33$ & $1.369(2)$ & $\mathrm{C} 19-\mathrm{C} 20$ & $1.368(2)$ \\
\hline S2-C6, S3-C13 & $1.7360(15), 1.7379(15)$ & S6-C30 & $1.7397(15)$ & $\mathrm{S} 1-\mathrm{C} 22$ & $1.7410(16)$ \\
\hline $\mathrm{S} 2-\mathrm{C} 9, \mathrm{~S} 3-\mathrm{C} 10$ & $1.7287(15), 1.7286(15)$ & S6-C33 & $1.7300(14)$ & S1-C19 & $1.7258(18)$ \\
\hline $\mathrm{C} 5-\mathrm{C} 6, \mathrm{C} 13-\mathrm{C} 14$ & $1.461(2), 1.462(2)$ & C29-C30 & $1.461(2)$ & $\mathrm{C} 23-\mathrm{C} 22$ & $1.456(2)$ \\
\hline C9-C10 & $1.454(2)$ & C33-C33A & $1.451(3)$ & C19-C19A & $1.450(3)$ \\
\hline Sil-C7 & $1.8888(15)$ & Si3-C31 & $1.8822(15)$ & B1-C21 & $1.567(2)$ \\
\hline \multirow[t]{5}{*}{ Si1-C22 } & $1.8702(17)$ & & & $\mathrm{B} 1-\mathrm{C} 1$ & $1.576(3)$ \\
\hline & & & & $\mathrm{B} 1-\mathrm{C} 10$ & $1.572(3)$ \\
\hline & & & & $\mathrm{C} 21-\mathrm{B}-\mathrm{C} 1$ & $116.18(15)$ \\
\hline & & & & $\mathrm{C} 1-\mathrm{B}-\mathrm{C} 10$ & $122.84(15)$ \\
\hline & & & & C10-B-C21 & $120.94(16)$ \\
\hline C8-C7-Si1/C11-C12-Si2 & $121.80(11) / 120.92(11)$ & C32-C31-Si3 & $120.72(11)$ & $\mathrm{C} 20-\mathrm{C} 21-\mathrm{B} 1$ & $118.99(16)$ \\
\hline C6-C7-Si1/C13-C12-Si2 & $127.78(11) / 128.44(11)$ & $\mathrm{C} 30-\mathrm{C} 31-\mathrm{Si} 3$ & $129.88(11)$ & $\mathrm{C} 22-\mathrm{C} 21-\mathrm{B} 1$ & $130.33(16)$ \\
\hline C7-C6-C5/C12-C13-C14 & $132.25(14) / 131.95(14)$ & C31-C30-C29 & $132.13(14)$ & $\mathrm{C} 21-\mathrm{C} 22-\mathrm{C} 23$ & $131.66(15)$ \\
\hline $\mathrm{C} 4-\mathrm{C} 5-\mathrm{C} 6 / \mathrm{C} 15-\mathrm{C} 14-\mathrm{C} 13$ & $127.73(14) / 127.44(14)$ & $\mathrm{C} 28-\mathrm{C} 29-\mathrm{C} 30$ & $127.48(14)$ & $\mathrm{C} 24-\mathrm{C} 23-\mathrm{C} 22$ & $130.92(16)$ \\
\hline Th1//Th2 & $5.87(8)$ & Th1//Th2 & 0.0 & Th1//Th2 & 0.0 \\
\hline Th2//Th3 & $45.13(5) / 47.30(5)$ & Th2//Th3 & $41.30(5)$ & Th2//Th3 & $15.16(9)$ \\
\hline
\end{tabular}

Figure S1. ORTEP plots of QT-SiMe ${ }_{3}(2$ molecules) and QT-BMes 2 (50\% thermal ellipsoids).
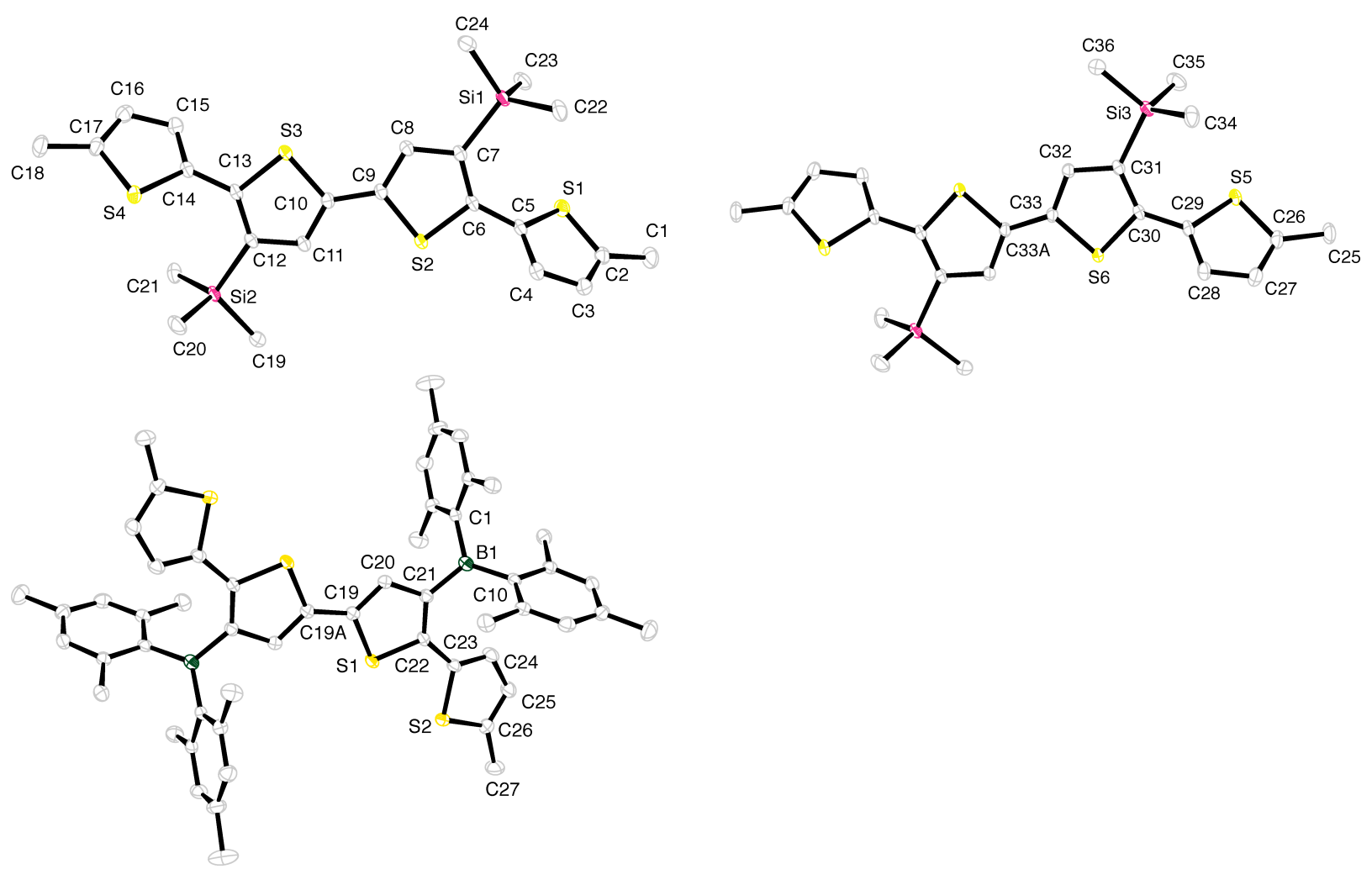
Table S2. Crystal data and structure refinement for QT-SiMe ${ }_{3}$ and QT-BMes ${ }_{2}$.

\begin{tabular}{|c|c|c|}
\hline Compound & QT-SiMe 3 & QT-BMes 2 \\
\hline Empirical formula & $\mathrm{C}_{24} \mathrm{H}_{30} \mathrm{~S}_{4} \mathrm{Si}_{2}$ & $\mathrm{C}_{55} \mathrm{H}_{57} \mathrm{~B}_{2} \mathrm{Cl}_{3} \mathrm{~S}_{4}$ \\
\hline$M$ & 502.90 & 974.22 \\
\hline$T, \mathrm{~K}$ & $100(2)$ & $100(2)$ \\
\hline Wavelength, ̊ & 1.54178 & 1.54178 \\
\hline crystal system & triclinic & Triclinic \\
\hline space group & $P_{1}$ & $P_{1}$ \\
\hline$a, \AA$ & $6.7152(3)$ & $8.34690(10)$ \\
\hline$b, \AA$ & $15.2227(7)$ & $11.5463(2)$ \\
\hline$c, \AA$ & $19.6615(9)$ & $13.6888(2)$ \\
\hline$\square$, deg & $89.651(2)$ & $100.7870(10)$ \\
\hline$\square$, deg & $88.840(2)$ & $90.2460(10)$ \\
\hline$\square \mathrm{deg}$ & $79.481(2)$ & $98.7110(10)$ \\
\hline$V, \AA^{3}$ & $1975.68(16)$ & $1280.28(3)$ \\
\hline$Z$ & 3 & 1 \\
\hline$\square$ calc, $\mathrm{g} \mathrm{cm}^{-3}$ & 1.268 & 3.411 \\
\hline$\square(\mathrm{MoK} \square), \mathrm{mm}^{-1}$ & 4.251 & 2.407 \\
\hline$F(000)$ & 798 & 512 \\
\hline crystal size, mm & $0.25 \times 0.12 \times 0.10$ & $0.15 \times 0.15 \times 0.04$ \\
\hline \multirow[t]{3}{*}{ limiting indices } & $-7<=\mathrm{h}<=7$ & $-9<=h<=9$ \\
\hline & $-17<=\mathrm{k}<=17$ & $-13<=\mathrm{k}<=13$ \\
\hline & $-23<=1<=22$ & $-16<=1<=16$ \\
\hline$\square$ range, deg & 2.95 to 64.97 & 3.29 to 65.12 \\
\hline reflns collected & 16423 & 22714 \\
\hline independent reflns & $6286[\mathrm{R}(\mathrm{int})=0.0204]$ & $4179[\mathrm{R}(\mathrm{int})=0.0299]$ \\
\hline Absorption correction & Semi-empirical from equivalents & Semi-empirical from equivalents \\
\hline Refinement method & Full-matrix least-squares on $F^{2}$ & Full-matrix least-squares on $F^{2}$ \\
\hline Data/ restraints / parameters & $6286 / 0 / 419$ & $4179 / 352 / 356$ \\
\hline Goodness-of-fit on $F^{2}$ & 1.045 & 1.029 \\
\hline Final $R$ indices $[\mathrm{I}>2 \square(\mathrm{I})]^{[\mathrm{a}]}$ & $\mathrm{R} 1=0.0260, \mathrm{wR} 2=0.0701$ & $\mathrm{R} 1=0.0365, \mathrm{wR} 2=0.0957$ \\
\hline $\mathrm{R}$ indices (all data) ${ }^{[\mathrm{a}]}$ & $\mathrm{R} 1=0.0278, \mathrm{wR} 2=0.0714$ & $\mathrm{R} 1=0.0404, \mathrm{wR} 2=0.0986$ \\
\hline peak/hole $\left(\mathrm{e} \AA^{-3}\right)$ & 0.361 and -0.220 & 0.371 and -0.258 \\
\hline
\end{tabular}

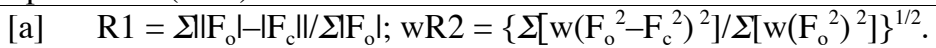




\section{Figure $S 2$.}

(a) Comparison of NMR spectra of QT-SiMe 3 and QT-BMes in $_{\mathbf{2}} \mathrm{CDCl}_{3}$.

\section{${ }^{1}$ H NMR:}

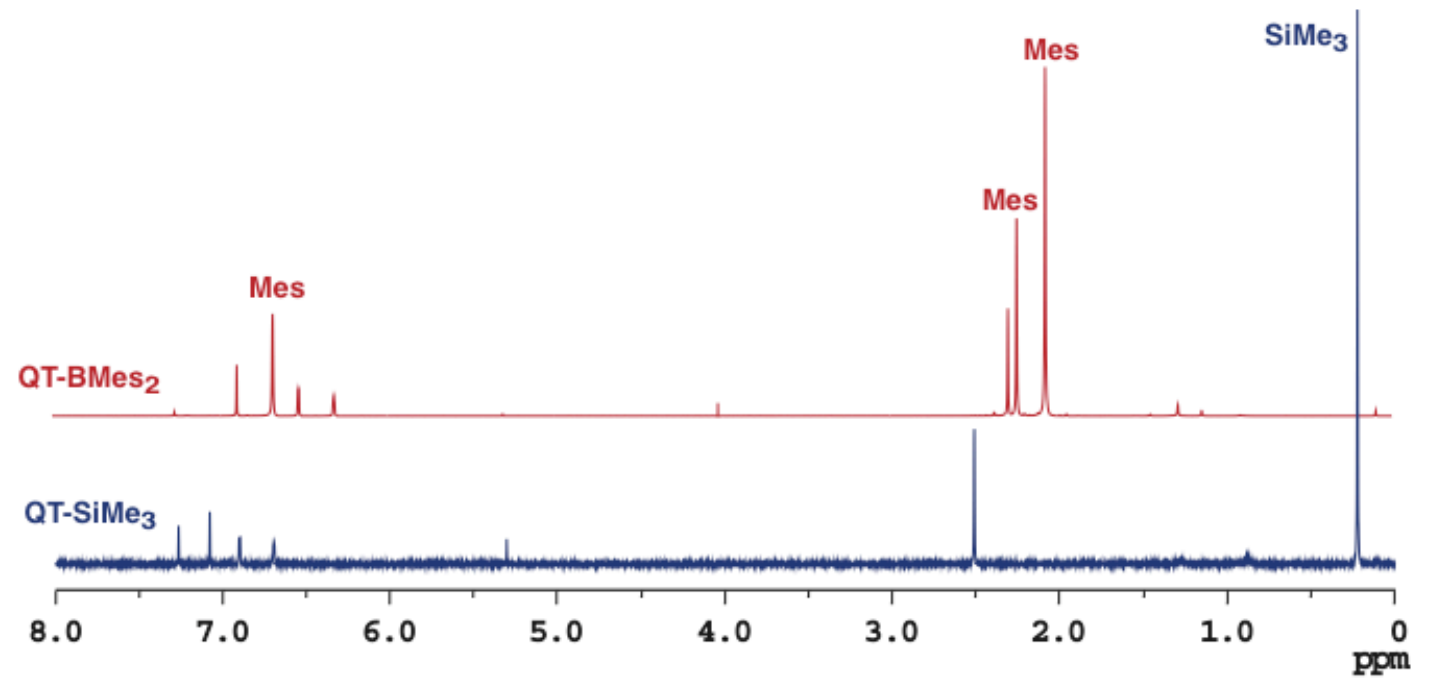

${ }^{13}$ C NMR (aromatic region):

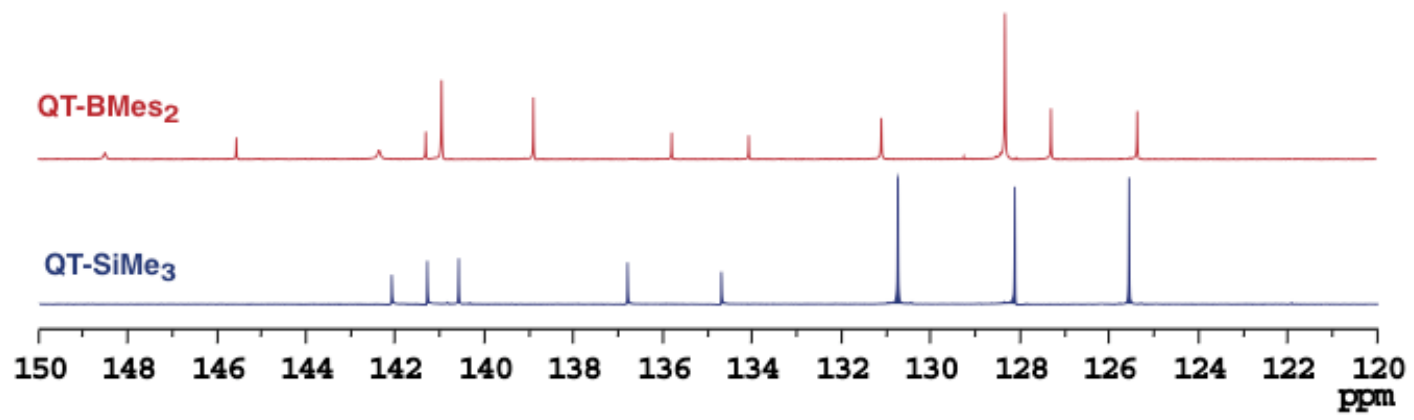


(b) Comparison of NMR spectra of PT-SiMe ${ }_{3}$ and PT-BMes 2 .

${ }^{1}$ H NMR:

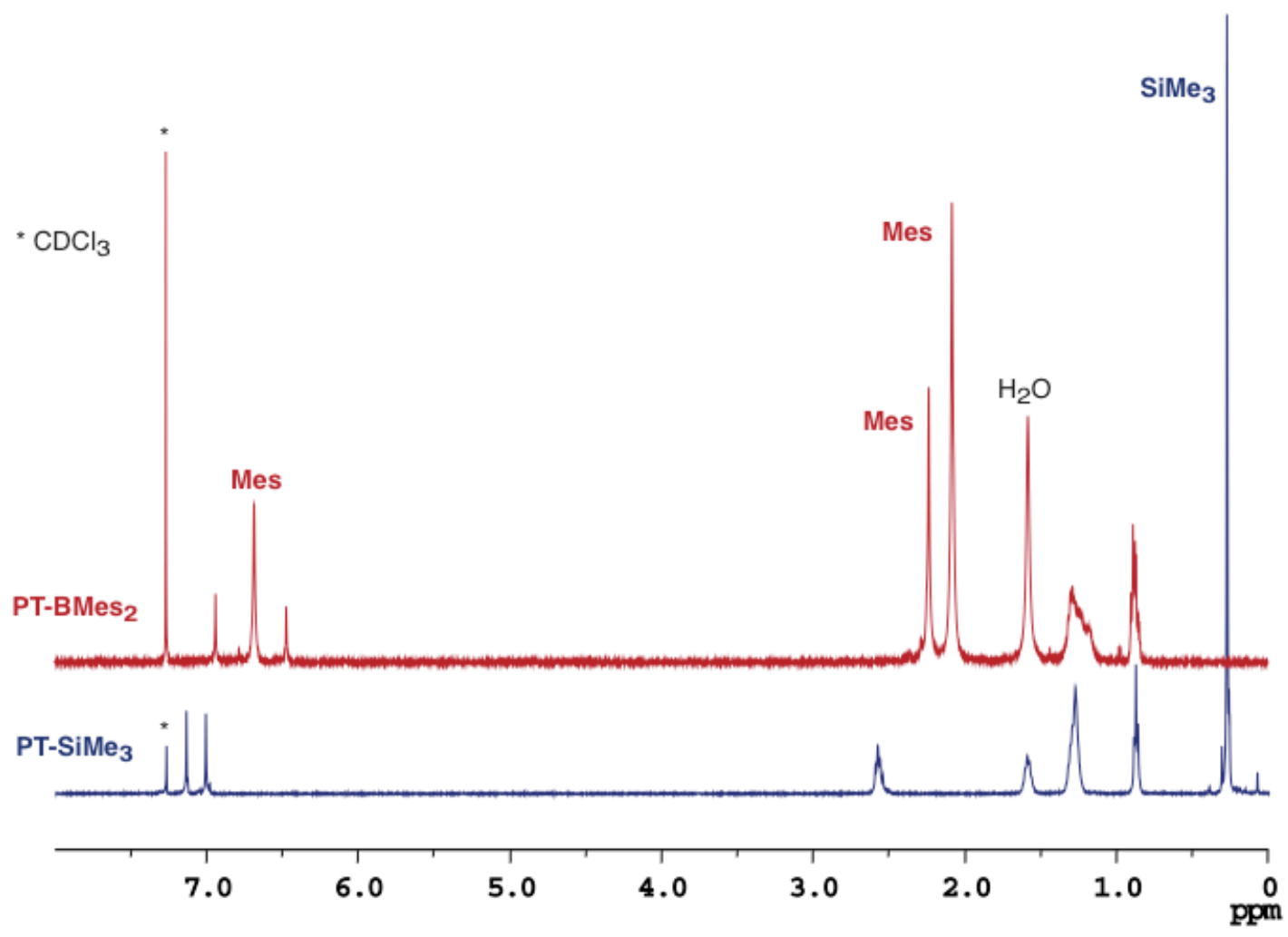

${ }^{13}$ C NMR:

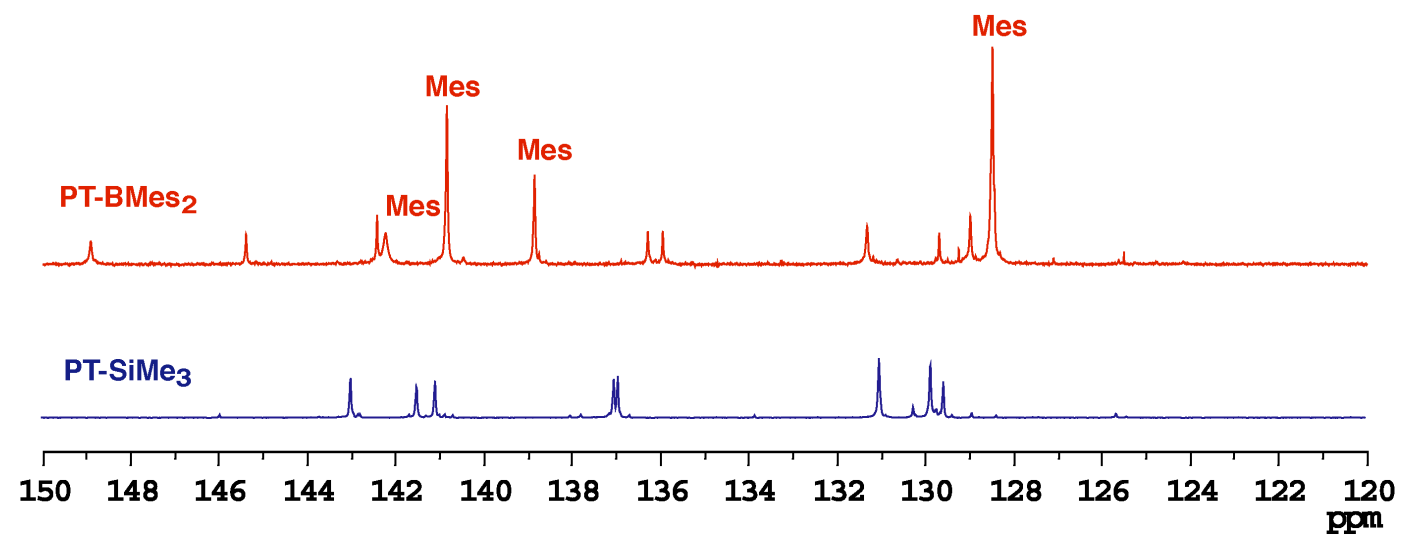




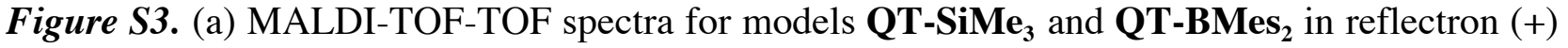
mode. (b) MALDI-TOF-TOF spectrum of PT-SiMe $\mathbf{S}_{3}$, showing two series of similar intensity corresponding to $\mathrm{H}-\left\{\mathrm{C}_{34} \mathrm{H}_{48} \mathrm{~S}_{4} \mathrm{Si}_{2}\right\}_{\mathrm{n}}-\mathrm{CH}_{3}$ and an overlay of $\mathrm{CH}_{3}-\mathrm{Th}_{2} \mathrm{Si}_{2}-\left(\mathrm{Th}_{2} \mathrm{Hx}_{2} \mathrm{Th}_{2} \mathrm{Si}_{2}\right)_{\mathrm{n}}-\mathrm{CH}_{3}$ / $\mathrm{H}-\left\{\mathrm{C}_{34} \mathrm{H}_{48} \mathrm{~S}_{4} \mathrm{Si}_{2}\right\}_{\mathrm{n}}-\mathrm{C}_{20} \mathrm{H}_{29} \mathrm{~S}_{2}$ in reflectron (+)mode; (c) MALDI-TOF spectrum of PT-BMes $\mathbf{B n}_{2}$ linear $(+)$ mode.

(a)

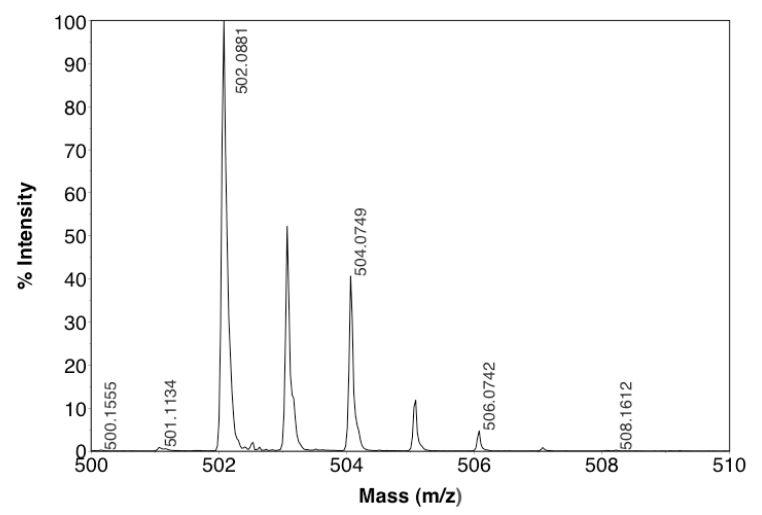

QT-BMes,

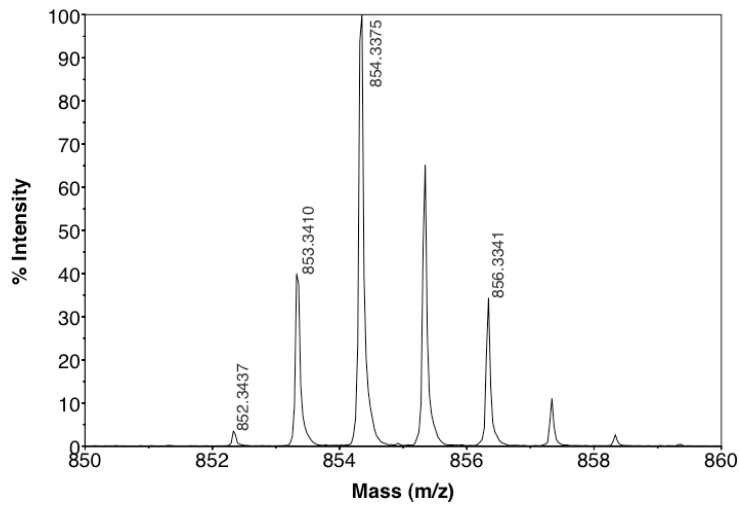

(b)

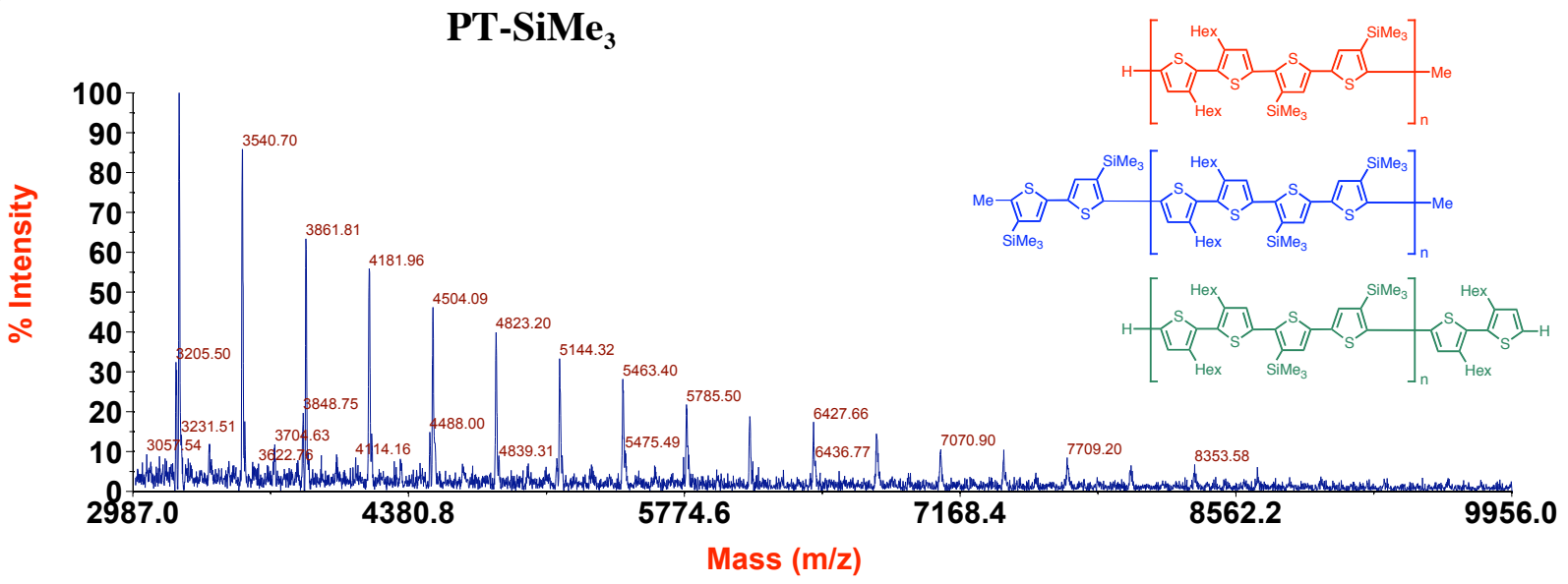

\begin{tabular}{|c|c|c|c|c|c|c|c|c|}
\hline Structure $/ n=$ & & 5 & 6 & 7 & 8 & 9 & 10 & 11 \\
\hline \multirow[t]{2}{*}{$\overline{\mathrm{H}-\left(\mathrm{Th}_{2} \mathrm{Hx}_{2} \mathrm{Th}_{2} \mathrm{Si}_{2}\right)_{\mathrm{n}}-\mathrm{CH}_{3}}$} & calcd & 3220 & 3861 & 4502 & $\overline{5143}$ & 5784 & 6425 & 7066 \\
\hline & observed & 3221.56 & 3861.81 & 4504.09 & 5144.32 & 5785.50 & 6427.66 & 7070.90 \\
\hline \multirow{2}{*}{ 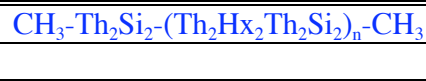 } & calcd & 3543 & 4184 & 4825 & 5466 & 6106 & $\begin{array}{c}6747 \\
\end{array}$ & 7388 \\
\hline & observed $^{[\mathrm{b}]}$ & 3540.70 & 4181.96 & 4823.20 & 5463.40 & 6105.58 & 6745.88 & 7389.11 \\
\hline \multirow[t]{2}{*}{ I $\mathrm{H}-\left(\mathrm{Th}_{2} \mathrm{Hx}_{2} \mathrm{Th}_{2} \mathrm{Si}_{2}\right)_{\mathrm{n}}-\mathrm{Th}_{2} \mathrm{Hx}_{2} \mathrm{H}$} & calcd & 3538 & 4179 & 4820 & 5461 & 6102 & 26743 & 7384 \\
\hline & observed $^{[b]}$ & 3540.70 & 4181.96 & 4823.20 & 5463.40 & 6105.58 & 6745.88 & 7389.11 \\
\hline
\end{tabular}

Notes: [a] Species containing Me end groups result from termination of the polymerization due to Me group transfer from the $\mathrm{Me}_{3} \mathrm{Sn}-\mathrm{Ar}-\mathrm{SnMe}_{3}$ species. [b] Overlap of the two sets of signals for $\mathrm{CH}_{3}-\mathrm{Th}_{2} \mathrm{Si}_{2}-\left(\mathrm{Th}_{2} \mathrm{Hx}_{2} \mathrm{Th}_{2} \mathrm{Si}_{2}\right)_{\mathrm{n}}-\mathrm{CH}_{3}$ and $\mathrm{H}-\left(\mathrm{Th}_{2} \mathrm{Hx}_{2} \mathrm{Th}_{2} \mathrm{Si}_{2}\right)_{\mathrm{n}}-$ $\mathrm{Th}_{2} \mathrm{Hx}_{2} \mathrm{H}$ leads to isotope patterns that corresponds to an overlay of the two and consequently the major peaks are between the calculated molecular ion peaks. 
(c)

\section{PT-BMes ${ }_{2}$}

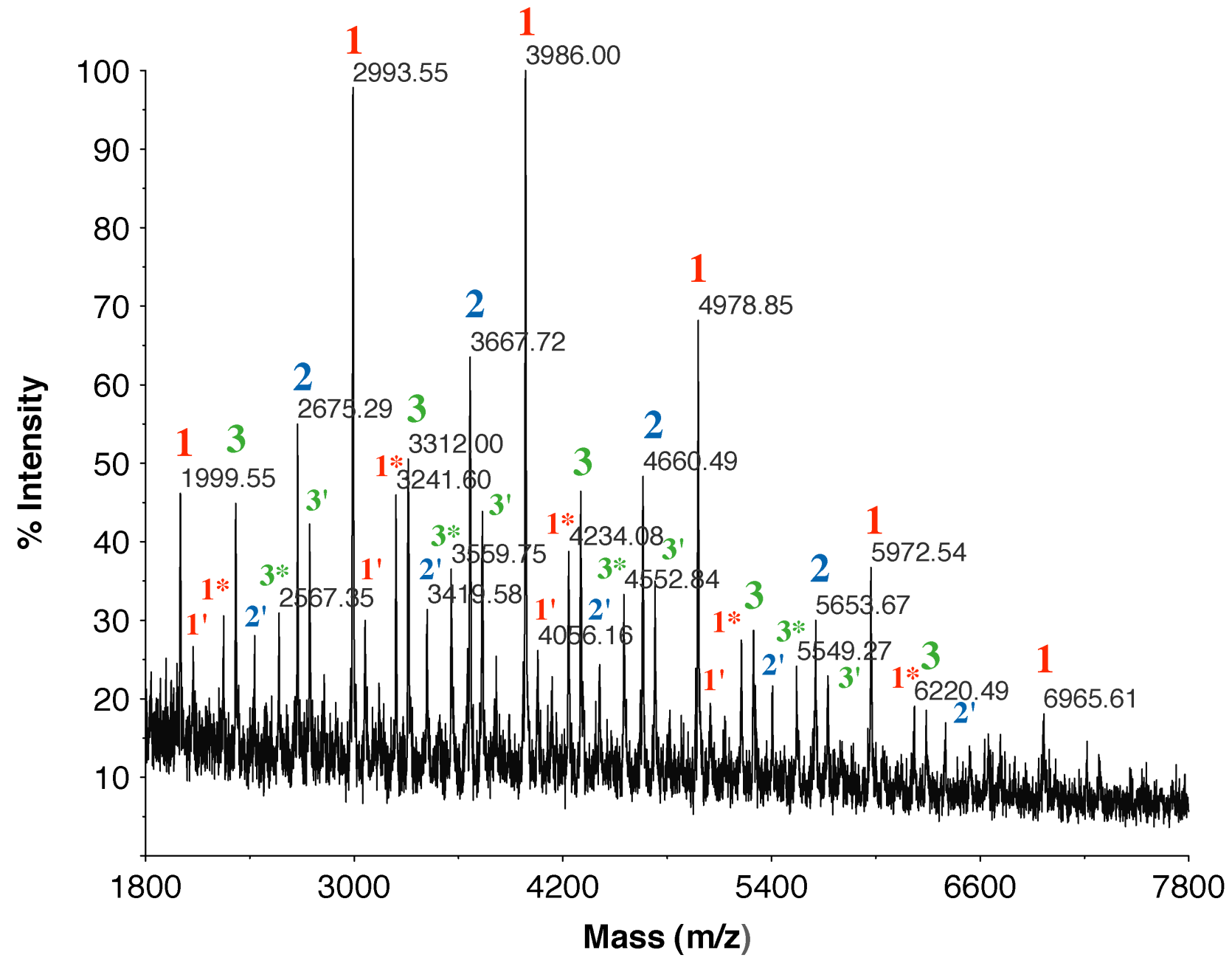

\begin{tabular}{|c|c|c|c|c|c|c|}
\hline Structure $/ n=$ & 2 & 2 & 3 & 4 & 5 & 6 \\
\hline \multirow[t]{2}{*}{$\mathrm{H}-\left(\mathrm{Th}_{2} \mathrm{Hx}_{2} \mathrm{Th}_{2} \mathrm{~B}_{2}\right)_{\mathrm{n}}-\mathrm{CH}_{3}(\mathbf{1})$} & calcd & 2001 & 2993 & 3986 & 4979 & 5972 \\
\hline & observed & 1999.55 & 2993.55 & 3986.00 & 4978.85 & 5972.54 \\
\hline \multirow[t]{2}{*}{$\mathrm{Mes}_{2} \mathrm{~B}-\left(\mathrm{Th}_{2} \mathrm{Hx}_{2} \mathrm{Th}_{2} \mathrm{~B}_{2}\right)_{\mathrm{n}}-\mathrm{CH}_{3}\left(1^{*}\right)$} & calcd & 2249 & 3241 & 4234 & 5227 & \\
\hline & observed & 2249.15 & 3241.40 & 4234.08 & 5227.39 & \\
\hline \multirow{2}{*}{$\begin{array}{l}\mathrm{H}-\left(\mathrm{Th}_{2} \mathrm{Hx}_{2} \mathrm{Th}_{2} \mathrm{~B}_{2}\right)_{\mathrm{n}}-\mathrm{CH}_{3}\left(1^{\prime}\right) \\
- \text { loss of } \mathrm{BMes}_{2}+\mathrm{H}\end{array}$} & calcd & & 2745 & 3738 & 4731 & 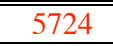 \\
\hline & observed & & 2745.35 & 3738.12 & 4730.71 & 5724.32 \\
\hline \multirow[t]{2}{*}{$\mathrm{CH}_{3}-\mathrm{Th}_{2} \mathrm{~B}_{2}-\left(\mathrm{Th}_{2} \mathrm{Hx}_{2} \mathrm{Th}_{2} \mathrm{~B}_{2}\right)_{\mathrm{n}}-\mathrm{CH}_{3}$} & calcd & 2675 & 3668 & 4660 & 5653 & \\
\hline & observed & 2675.29 & 3667.72 & 4660.49 & 5653.67 & \\
\hline \multirow{2}{*}{$\begin{array}{l}\mathrm{CH}_{3}-\mathrm{Th}_{2} \mathrm{~B}_{2}-\left(\mathrm{Th}_{2} \mathrm{Hx}_{2} \mathrm{Th}_{2} \mathrm{~B}_{2}\right)_{\mathrm{n}}-\mathrm{CH}_{3}\left(2^{\prime}\right) \\
- \text { loss of } \mathrm{BMes}_{2}+\mathrm{H} \text { calcd }\end{array}$} & calcd & 2427 & 3420 & 4412 & 5405 & \\
\hline & observed & 2426.75 & 3419.58 & 4412.12 & 5406.13 & \\
\hline \multirow[t]{2}{*}{$\mathrm{H}-\left(\mathrm{Th}_{2} \mathrm{Hx}_{2} \mathrm{Th}_{2} \mathrm{~B}_{2}\right)_{\mathrm{n}}-\mathrm{Th}_{2} \mathrm{Hx}_{2} \mathrm{H}(3)$} & calcd & 2319 & 3312 & 4305 & 5297 & 6290 \\
\hline & observed & 2319.24 & 3312.00 & 4304.33 & 5297.18 & 6290.86 \\
\hline \multirow[t]{2}{*}{$\mathrm{Mes}_{2} \mathrm{~B}-\left(\mathrm{Th}_{2} \mathrm{Hx}_{2} \mathrm{Th}_{2} \mathrm{~B}_{2}\right)_{\mathrm{n}}-\mathrm{Th}_{2} \mathrm{Hx}_{2} \mathrm{H}\left(3^{*}\right)$} & calcd & 2567 & 3560 & 4553 & & \\
\hline & observed & 2567.35 & 3559.75 & 4552.84 & & \\
\hline \multirow{2}{*}{$\begin{array}{l}\mathrm{H}-\left(\mathrm{Th}_{2} \mathrm{Hx}_{2} \mathrm{Th}_{2} \mathrm{~B}_{2}\right)_{\mathrm{n}}-\mathrm{Th}_{2} \mathrm{Hx}_{2} \mathrm{H}\left(3^{\prime}\right) \\
- \text { loss of } \mathrm{BMes}_{2}+\mathrm{H}\end{array}$} & calcd & 2071 & 3064 & 4057 & 5049 & \\
\hline & observed & 2075.50 & 3063.80 & 4056.16 & 5048.18 & \\
\hline
\end{tabular}

Notes: Species marked with * result from borylation of the $\mathrm{Th}_{2} \mathrm{Hx}_{2}$ end group; species marked with ' result from desilylation due to generation of small quantities of $\mathrm{HBr}$. 
Figure S4. Absorption and emission spectra for polymers PT-SiMe $\mathbf{S}_{3}$ and PT-BMes 2 in comparison to the model compounds QT-SiMe 3 and QT-BMes 2 .
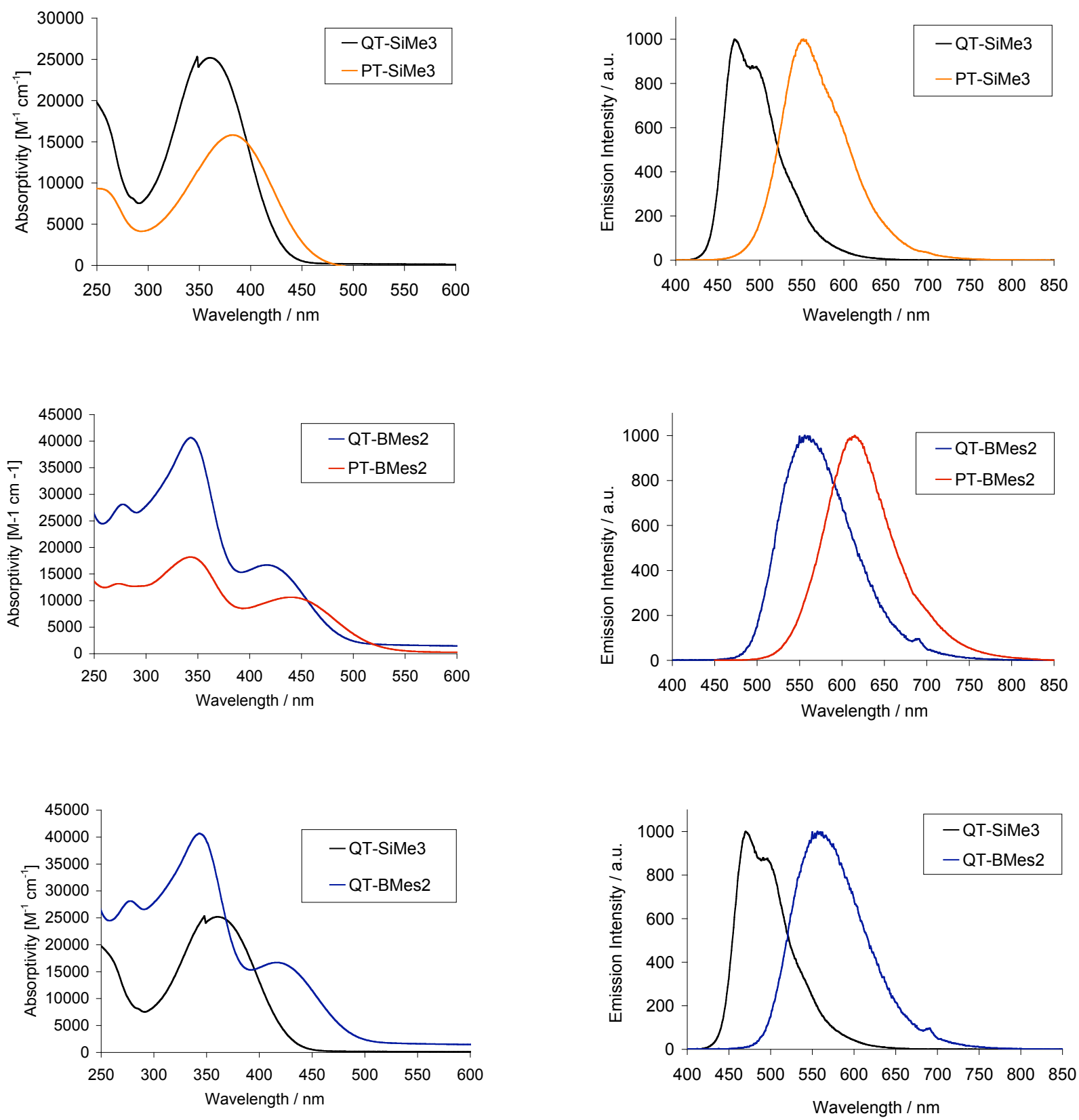
Figure S5. Computed (Gaussian03) orbital plots for QT-SiMe 3 and QT-BMes . $_{\mathbf{3}}$

QT-SiMe $_{3} \quad$ QT-BMes $_{2}$

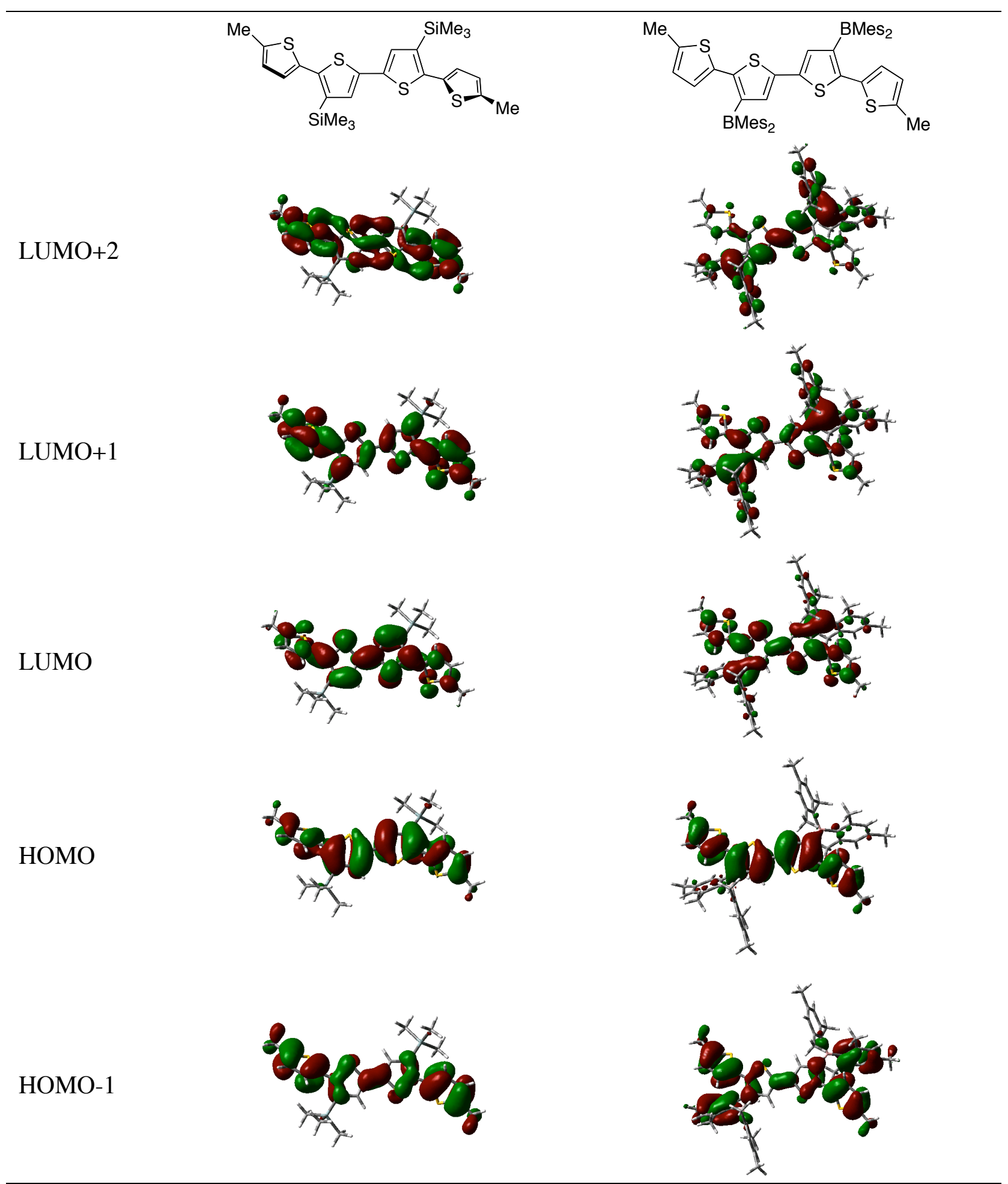


Table S3. Calculated orbital energies (eV) for QT-SiMe $\mathbf{S}_{3}$ and $\mathbf{Q T}-\mathbf{B M e s}_{2}$ from DFT (B3LYP) calculations.

\begin{tabular}{|c|c|c|c|c|}
\hline \multicolumn{2}{|c|}{ Compound } & QT-SiMe $_{3}$ & \multicolumn{2}{|c|}{$\mathrm{QT}^{-B M e s}{ }_{2}$} \\
\hline \multicolumn{2}{|c|}{ LUMO+2 } & $\begin{array}{c}-0.299 \\
(\mathrm{QT})\end{array}$ & \multicolumn{2}{|c|}{$\begin{array}{c}-1.143 \\
(\text { Mes-B-Th } \\
\text {-B-Mes })\end{array}$} \\
\hline \multicolumn{2}{|c|}{ LUMO+1 } & $\begin{array}{c}-0.707 \\
\text { (outer Th) }\end{array}$ & \multicolumn{2}{|c|}{$\begin{array}{c}-1.687 \\
\text { (B) }\end{array}$} \\
\hline \multicolumn{2}{|c|}{ LUMO } & $\begin{array}{c}-1.524 \\
(\mathrm{QT})\end{array}$ & \multicolumn{2}{|c|}{-1.850} \\
\hline \multicolumn{2}{|c|}{ HOMO } & $\begin{array}{c}-5.034 \\
(\mathbf{Q T})\end{array}$ & \multicolumn{2}{|c|}{$\begin{array}{l}-4.952 \\
(\mathbf{Q T})\end{array}$} \\
\hline \multicolumn{2}{|c|}{ HOMO-1 } & $\begin{array}{l}-5.932 \\
(\mathrm{QT})\end{array}$ & \multicolumn{2}{|c|}{$\begin{array}{c}-\mathbf{5 . 6 8 7} \\
\text { (outer Th, Mes) }\end{array}$} \\
\hline \multicolumn{2}{|c|}{ HOMO-LUMO gap } & 3.510 & \multicolumn{2}{|c|}{3.102} \\
\hline \multicolumn{5}{|c|}{$\begin{array}{l}\text { QT }=\text { quaterthiophene moiety; the orbitals that are involved in the computed lowest energy } \\
\text { transitions are marked in red and blue. }\end{array}$} \\
\hline \multicolumn{5}{|c|}{$\begin{array}{l}\text { Table S4. Calculated electronic transitions for QT-SiMe } \mathbf{Q}_{3} \text { and QT-BMes } \text { from TD-DFT (B3LYP) }_{\text {Q }} \text { (Blculations. }\end{array}$} \\
\hline Compound & Transition & MO contributions & $\begin{array}{l}\text { Energy gap } \\
\mathrm{eV}(\mathrm{nm})\end{array}$ & $\begin{array}{l}\text { Oscillator } \\
\text { strength / } \mathrm{f}\end{array}$ \\
\hline $\mathrm{QT}_{-\mathrm{SiMe}_{3}}$ & $\mathbf{S}_{0} \rightarrow \mathbf{S}_{1}$ & HOMO $\rightarrow$ LUMO & $3.1764(390)$ & 1.0069 \\
\hline \multirow[t]{5}{*}{ QT-BMes 2} & $\mathbf{S}_{0} \rightarrow \mathbf{S}_{1}$ & $\begin{array}{l}\text { HOMO } \rightarrow \text { LUMO } \quad(\mathbf{0 . 6 5 1 6 2}) \\
\mathrm{HOMO} \rightarrow \text { LUMO+2 }(0.11494)\end{array}$ & 2.7033 (459) & 0.3731 \\
\hline & $\mathrm{S}_{0} \rightarrow \mathrm{S}_{2}$ & $\mathrm{HOMO} \rightarrow \mathrm{LUMO}+1 \quad(0.67681)$ & $2.7487(451)$ & 0.0051 \\
\hline & $\mathbf{S}_{\mathbf{0}} \rightarrow \mathrm{S}_{3}$ & HOMO-1 $\rightarrow$ LUMO+1 & $3.2849(377)$ & 0.7555 \\
\hline & & HOMO $\rightarrow$ LUMO $\quad(-0.10490)$ & & \\
\hline & & HOMO $\rightarrow$ LUMO+2 (0.59056) & & \\
\hline
\end{tabular}

